\title{
A Rigidity Result for a Reduced Model of a Cubic-to-Orthorhombic Phase Transition in the Geometrically Linear Theory of Elasticity*
}

\author{
Angkana Rüland ${ }^{\dagger}$
}

July 22, 2018

\begin{abstract}
We study a simplified two-dimensional model for a cubic-to-orthorhombic phase transition occuring in certain shape-memory-alloys. In the low temperature regime the linear theory of elasticity predicts various possible patterns of martensite arrangements: Apart from the well known laminates this phase transition displays additional structures involving four martensitic variants - so called crossing twins.

Introducing a variational model including surface energy, we show that these structures are rigid under small energy perturbations. Combined with an upper bound construction this gives the optimal scaling behavior of incompatible microstructures. These results are related to papers by Capella and Otto, 2], 3], as well as to a paper by Dolzmann and Müller, 4 .
\end{abstract}

\section{Contents}

1 Introduction

1.1 The Stress-free Setting . . . . . . . . . . . . . . . . . . . . 2

1.2 The Setting of Small Deviations from the Stress-free Situation . . . . . . 4

2 The Proofs

2.1 Rigidity of the Outer Structure . . . . . . . . . . . . . 8

2.2 Proof of the Second Result _ . . . . . . . . . . . . . 15

2.3 Proof of the Third Statement . . . . . . . . . . . . . . . 23

${ }^{*}$ This work was part of the diploma thesis of the author written under the supervision of Prof. Felix Otto. She is greatly indebted to him for introducing her to the topic, insightful discussions, comments and advice. Further, she would like to thank her PhD advisor Prof. Herbert Koch for his constant support and advice. She thanks the Deutsche Telekomstiftung and the Hausdorff Center for Mathematics for financial support and the Max-Planck-Institute for Mathematics in the Sciences in Leipzig for its kind hospitality.

${ }^{\dagger}$ Mathematisches Institut, Universität Bonn, Endenicher Allee 60, 53115 Bonn, Germany 
2.4 Optimality and Branching . . . . . . . . . . . . . 31

\section{Introduction}

\subsection{The Stress-free Setting}

Working in the framework of linear elasticity, six stress-free strains characterize the body-centered to face-centered cubic-to-orthorhombic phase transition:

$$
\begin{aligned}
& e^{(1)}=\epsilon\left(\begin{array}{rrr}
1 & \delta & 0 \\
\delta & 1 & 0 \\
0 & 0 & -2
\end{array}\right), e^{(2)}=\epsilon\left(\begin{array}{rrr}
1 & -\delta & 0 \\
-\delta & 1 & 0 \\
0 & 0 & -2
\end{array}\right), \\
& e^{(3)}=\epsilon\left(\begin{array}{rrr}
1 & 0 & \delta \\
0 & -2 & 0 \\
\delta & 0 & 1
\end{array}\right), e^{(4)}=\epsilon\left(\begin{array}{rrr}
1 & 0 & -\delta \\
0 & -2 & 0 \\
-\delta & 0 & 1
\end{array}\right), \\
& e^{(5)}=\epsilon\left(\begin{array}{rrr}
-2 & 0 & 0 \\
0 & 1 & \delta \\
0 & \delta & 1
\end{array}\right), e^{(6)}=\epsilon\left(\begin{array}{rrr}
-2 & 0 & 0 \\
0 & 1 & -\delta \\
0 & -\delta & 1
\end{array}\right) .
\end{aligned}
$$

Here $\epsilon$ and $\delta$ are dimensionless parameters of typical magnitude $\sim 0.01$ and $\sim 0.25$, respectively. Stress-free configurations of phases are therefore solutions to the 6 -well problem:

$$
e(u) \in\left\{e^{(1)}, \ldots, e^{(6)}\right\},
$$

where $e(u)=\frac{\nabla u+(\nabla u)^{t}}{2}$ is the strain tensor describing the relative changes of length and $u$ is a displacement field indicating how much a particle has been moved under the deformation.

In contrast to the cubic-to-tetragonal phase transition for which Dolzmann and Müller, 4, proved that (locally) only simple laminates occur, experiments suggest that in the cubic-to-orthorhombic phase transition crossing twin structures, i.e. structures involving zig-zag-bands of four martensitic phases (c.f. Figure 1), have to be expected. In order to capture these configurations we consider a simplified model: We assume that the strains are two-dimensional and only four variants of martensite, say variant one to four, are present. Carrying out calculations in the piecewise affine setting, we observe that there are exactly two twinning connections between any of the martensites and that there exist precisely six martensitic crossing twin structures involving planar four-fold corners. These four-fold corners can be iterated to form the crossing twin structures.

In this setting it proves to be advantageous to carry out a change of coordinates and 


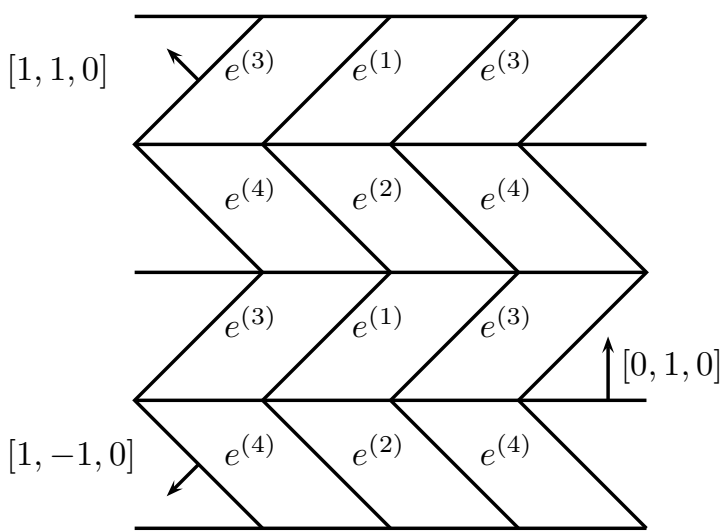

(a)

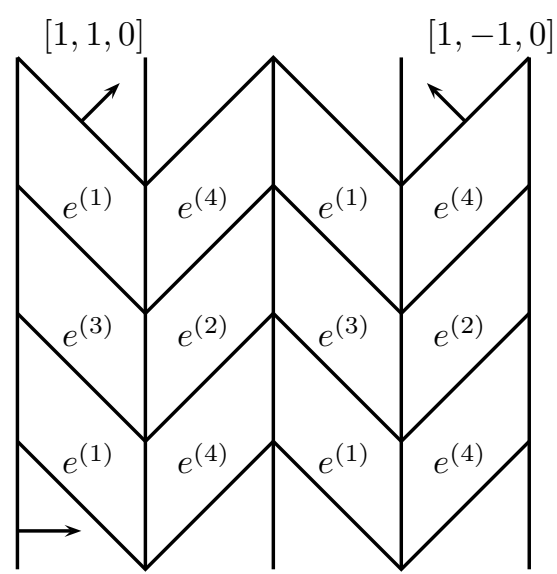

$[1,0,0]$

(b)

Figure 1: Possible crossing twin structures in the $y_{1}, y_{2}$-plane: (a) corresponds to $e_{12}=$ $e_{12}\left(y_{2}\right)$, (b) corresponds to $e_{12}=e_{12}\left(y_{1}\right)$. All possible crossing twin configurations of the simplified setting display a characteristic structure: They consist of doublelaminates which are made of an "outer structure" - here given by pairs of variants 1 and 3 , as well as 2 and 4 - and an "inner structure" determining the precise arrangement of the phases - here these are given by the twinning modes of the pairs 1 and 3 and 2 and 4, respectively. One notices that the relative volume fractions $\theta_{i}$ are determined by these structures: In (a) we have $\frac{\theta_{1}}{\theta_{1}+\theta_{3}}=\frac{\theta_{2}}{\theta_{2}+\theta_{4}}$, whereas in (b) the situation is described by $\frac{\theta_{1}}{\theta_{1}+\theta_{3}}=\frac{\theta_{4}}{\theta_{2}+\theta_{4}}$.

to renormalize the strains: Setting

$$
C=\frac{1}{\sqrt{2}}\left(\begin{array}{ccc}
0 & 1 & 1 \\
\sqrt{2} & 0 & 0 \\
0 & 1 & -1
\end{array}\right) \cdot \sqrt{6} \delta\left(\begin{array}{ccc}
\frac{1}{\sqrt{3}} & 0 & 0 \\
0 & \frac{\sqrt{3}}{\sqrt{2} \delta} & 0 \\
0 & 0 & \frac{1}{\sqrt{3}}
\end{array}\right)
$$

we are left with the following matrices

$$
\begin{aligned}
& \tilde{e}^{(1)}=\frac{\epsilon}{2 d}\left(\begin{array}{rrr}
d_{1} & 1 & 1 \\
1 & d_{2} & 1 \\
1 & 1 & d_{3}
\end{array}\right), \quad \tilde{e}^{(2)}=\frac{\epsilon}{2 d}\left(\begin{array}{rrr}
d_{1} & -1 & 1 \\
-1 & d_{2} & -1 \\
1 & -1 & d_{3}
\end{array}\right), \\
& \tilde{e}^{(3)}=\frac{\epsilon}{2 d}\left(\begin{array}{rrr}
d_{1} & 1 & -1 \\
1 & d_{2} & -1 \\
-1 & -1 & d_{3}
\end{array}\right), \quad \tilde{e}^{(4)}=\frac{\epsilon}{2 d}\left(\begin{array}{rrr}
d_{1} & -1 & -1 \\
-1 & d_{2} & 1 \\
-1 & 1 & d_{3}
\end{array}\right),
\end{aligned}
$$

where $d^{-1}=6 \delta^{2}, d_{1}=-\frac{1}{3}, d_{2}=\frac{3}{2 \delta^{2}}, d_{3}=-\frac{1}{3}$. In the sequel we will suppress the tildes in the notation. In these coordinates the first four strain tensors are highly 
symmetric, therefore if we are interested in two dimensional strains depending on only two coordinate directions, we can w.l.o.g. consider configurations depending on the $y_{1}, y_{2}$-coordinates only. For these it is possible to show that generic configurations are given by crossing twin phase distributions. The main result of the stress-free setting is formulated in the following theorem (c.f. [13]):

Theorem 1. Let $U \subset \mathbb{R}^{3}$ be open, convex. Assume $e \in \operatorname{Sym}(3, \mathbb{R}), e=e\left(y_{1}, y_{2}\right)=$ $\frac{\nabla u+(\nabla u)^{t}}{2}, u \in W^{1, \infty}\left(U, \mathbb{R}^{3}\right)$ such that $e(u) \in\left\{e^{(1)}, e^{(2)}, e^{(3)}, e^{(4)}\right\}$ in $U$.

1. Then the following dichotomy holds:

$$
e_{12}=e_{12}\left(y_{1}\right) \text { or } e_{12}=e_{12}\left(y_{2}\right)
$$

2. In case $e_{12}=e_{12}\left(y_{1}\right)$ there exists a function $g(t)$ such that:

$$
\left(e_{13} \circ \Phi\right)(s, t)=e_{12}(s) g(t) \text { and }\left(e_{23} \circ \Phi\right)(s, t)=g(t) \text {, }
$$

where $\Phi(s, t)=\left(s,-E_{12}(s)+t\right)$ and $E_{12}^{\prime}\left(y_{1}\right)=e_{12}\left(y_{1}\right), E_{12}(0)=0$. Due to symmetry, $e=e\left(y_{1}, y_{2}\right)$ can also be replaced by $e\left(y_{1}, y_{3}\right)$ and $e\left(y_{2}, y_{3}\right)$ respectively which yields analogous results.

In this model involving only four (two-dimensional) strains, we do not have to require additional BV-regularity for the strain tensors.

\subsection{The Setting of Small Deviations from the Stress-free Situ- ation}

In the present paper we are interested in the rigidity of these constructions. In the spirit of the papers [2], 3] of Capella and Otto, we introduce a variational model consisting of an elastic and a surface energy contribution rescaled in an optimal manner. More precisely, we use an elastic energy of the form

$$
E_{\text {elast }}=2 \mu \int_{B_{L}}\left|e-\chi_{1} e^{(1)}-\chi_{2} e^{(2)}-\chi_{3} e^{(3)}-\chi_{4} e^{(4)}\right|^{2} d y,
$$

where $\chi_{k}$ are the characteristic functions of the martensitic phases, i.e. $\chi_{k} \in\{0,1\}, \mu$ is a material constant - the first Lamé-constant - of the dimension $\frac{J}{m^{3}}$, and $B_{L} \subset \mathbb{R}^{3}$ denotes the sample. As we are dealing with the analogue of the stress-free setting, we will assume that all quantities involved only depend on the $y_{1}, y_{2}$ variables. The elastic energy can be rewritten in terms of the modified characteristic functions $\tilde{\chi}_{k}$ which are defined as

$$
\begin{aligned}
& \tilde{\chi}_{1}=1-2\left(\chi_{2}+\chi_{3}\right), \\
& \tilde{\chi}_{2}=1-2\left(\chi_{3}+\chi_{4}\right), \\
& \tilde{\chi}_{3}=1-2\left(\chi_{2}+\chi_{4}\right), \\
& \chi_{1}+\chi_{2}+\chi_{3}+\chi_{4}=1, \quad \chi_{k} \in\{0,1\}, \quad \tilde{\chi}_{k} \in\{-1,1\} .
\end{aligned}
$$


With these the elastic energy takes the form:

$$
E_{\text {elast }}(e)=2 \mu \int_{B_{L}}\left|e-\frac{\epsilon}{2 d}\left(\begin{array}{ccc}
d_{1} & \tilde{\chi}_{3} & \tilde{\chi}_{2} \\
\tilde{\chi}_{3} & d_{2} & \tilde{\chi}_{1} \\
\tilde{\chi}_{2} & \tilde{\chi}_{1} & d_{3}
\end{array}\right)\right|^{2} d y, e=e(u)\left(y_{1}, y_{2}\right) \text {, }
$$

where $d, d_{1}, d_{2}, d_{3}$ are the constants obtained from the change of coordinates in the stress-free setting.

As it is well known that this energy is not weakly lower semicontinuous, we cannot hope to prove rigidity in this framework. Thus, a surface energy punishing high oscillations is introduced:

$$
E_{\text {surf }}=\kappa \int_{B_{L}}\left|\nabla \chi_{1}\right|+\left|\nabla \chi_{2}\right|+\left|\nabla \chi_{3}\right|+\left|\nabla \chi_{4}\right| d y
$$

Here $\kappa$ is a further material parameter of the units $\frac{J}{m^{2}}$. Working with these two energy contributions it is known that there are two regimes for incompatible microstructures. In 9, Kohn and Müller point out that the choice of the regime depends on a single non-dimensional quantity: $\eta:=\frac{2 d^{2} \kappa}{\epsilon^{2} \mu L}$. On the one hand, if $\eta \gg 1$, low-energy incompatible microstructures are characterized by fine-scale oscillations of twins and an energy contribution scaling as $E \sim\left(\kappa L^{2}\right)^{\frac{1}{2}}\left(2 \frac{\epsilon^{2}}{4 d^{2}} \mu L^{3}\right)^{\frac{1}{2}}$. If on the other hand $\eta \ll 1$, branching is energetically preferred and yields a scaling behavior of $E \sim\left(\kappa L^{2}\right)^{\frac{2}{3}}\left(2 \frac{\epsilon^{2}}{4 d^{2}} L^{3} \mu\right)^{\frac{1}{3}}$. Rescaling all quantities by their natural units and energy so as to capture the regime of $\eta \ll 1$, we have:

$$
\begin{aligned}
& y=L \hat{y}, \\
& e=\frac{\epsilon}{2 d} \hat{e}, \\
& E_{\text {elast }}=\frac{\epsilon^{2} L^{3} \mu}{2 d^{2}} \hat{E}_{\text {elast }}, \\
& E_{\text {surf }}=\kappa L^{2} \hat{E}_{\text {surf }}, \\
& E=\left(\frac{2 d^{2} \kappa}{\epsilon^{2} \mu L}\right)^{\frac{2}{3}} \frac{\epsilon^{2}}{2 d^{2}} L^{3} \mu \hat{E} .
\end{aligned}
$$

With this we can point out the relevant quantities in their non-dimensional versions:

Definition 1. We are interested in

- the strains $e=\frac{\nabla u+(\nabla u)^{t}}{2}$, where $u: \mathbb{R}^{3} \rightarrow \mathbb{R}^{3}, u \in W^{1, \infty}\left(\mathbb{R}^{3}, \mathbb{R}^{3}\right)$ and $e^{(i)}=$ 
$e^{(i)}\left(y_{1}, y_{2}\right)$,

$$
\begin{aligned}
& e^{(1)}=\frac{\epsilon}{2 d}\left(\begin{array}{ccc}
d_{1} & 1 & 1 \\
1 & d_{2} & 1 \\
1 & 1 & d_{3}
\end{array}\right), e^{(2)}=\frac{\epsilon}{2 d}\left(\begin{array}{rrr}
d_{1} & -1 & 1 \\
-1 & d_{2} & -1 \\
1 & -1 & d_{3}
\end{array}\right), \\
& e^{(3)}=\frac{\epsilon}{2 d}\left(\begin{array}{ccc}
d_{1} & 1 & -1 \\
1 & d_{2} & -1 \\
-1 & -1 & d_{3}
\end{array}\right), e^{(4)}=\frac{\epsilon}{2 d}\left(\begin{array}{rrr}
d_{1} & -1 & -1 \\
-1 & d_{2} & 1 \\
-1 & 1 & d_{3}
\end{array}\right),
\end{aligned}
$$

where $d^{-1}=6 \delta^{2}, d_{1}=-\frac{1}{3}, d_{2}=\frac{3}{2 \delta^{2}}, d_{3}=-\frac{1}{3}$,

- the characteristic functions $\chi_{i}\left(y_{1}, y_{2}\right) \in\{0,1\}, i \in\{1, \cdots, 4\}$, with $\chi_{1}+\chi_{2}+$ $\chi_{3}+\chi_{4}=1$ for the martensite phases,

- the modified characteristic functions

$$
\begin{aligned}
& \tilde{\chi}_{1}=1-2\left(\chi_{2}+\chi_{3}\right), \\
& \tilde{\chi}_{2}=1-2\left(\chi_{3}+\chi_{4}\right), \\
& \tilde{\chi}_{3}=1-2\left(\chi_{2}+\chi_{4}\right), \\
& \tilde{\chi}_{i} \in\{-1,1\} .
\end{aligned}
$$

These can be reformulated in terms of the original characteristic functions:

$$
\begin{aligned}
& \chi_{1}=\frac{1}{4}\left(1+\tilde{\chi}_{2}+\tilde{\chi}_{3}+\tilde{\chi}_{1}\right), \\
& \chi_{2}=\frac{1}{4}\left(1+\tilde{\chi}_{2}-\tilde{\chi}_{3}-\tilde{\chi}_{1}\right), \\
& \chi_{3}=\frac{1}{4}\left(1-\tilde{\chi}_{2}+\tilde{\chi}_{3}-\tilde{\chi}_{1}\right), \\
& \chi_{4}=\frac{1}{4}\left(1-\tilde{\chi}_{2}-\tilde{\chi}_{3}+\tilde{\chi}_{1}\right),
\end{aligned}
$$

- the parameter $\eta:=\frac{2 d^{2} \kappa}{\epsilon^{2} \mu L}$,

- the elastic energy

$$
E_{\text {elast }}=\int_{B_{1}}\left|e-\left(\begin{array}{ccc}
d_{1} & \tilde{\chi}_{3} & \tilde{\chi}_{2} \\
\tilde{\chi}_{3} & d_{2} & \tilde{\chi}_{1} \\
\tilde{\chi}_{2} & \tilde{\chi}_{1} & d_{3}
\end{array}\right)\right|^{2} d y, e=e\left(y_{1}, y_{2}\right),
$$

- the surface energy

$$
E_{\text {surf }}=\int_{B_{1}}\left(\left|\nabla \chi_{1}\right|+\left|\nabla \chi_{2}\right|+\left|\nabla \chi_{3}\right|+\left|\nabla \chi_{4}\right|\right) d y,
$$


- the total energy

$$
E:=\eta^{\frac{1}{3}} E_{\text {surf }}+\eta^{-\frac{2}{3}} E_{\text {elast }} .
$$

We will always suppose $E \leq 1$ in the sequel. If we want to stress the dependence on $\eta, \chi$ or e we will also use the notation $E_{\eta}(e, \chi)$.

We remark that one should think of $\tilde{\chi}_{1}, \tilde{\chi}_{2}, \tilde{\chi}_{3}$ as corresponding to $e_{23}, e_{13}, e_{12}$. Mimicking the proof of the stress-free case, c.f. [13], we show that the configurations are rigid - at least in a weak sense:

Theorem 2. 1. Let $\tilde{\chi}_{i}, E$ be as in Definition 1, let $\eta \leq 1$. Then there exists $r>0$ and functions $f_{(100)}, f_{(010)}$, such that:

$$
\int_{B_{r}}\left|\tilde{\chi}_{3}-f_{(100)}\right| d x \lesssim E^{\frac{1}{2}} \text { or } \int_{B_{r}}\left|\tilde{\chi}_{3}-f_{(010)}\right| d x \lesssim E^{\frac{1}{2}}
$$

where $f_{(100)}=f\left(y_{1}\right) \in\{-1,1\}, f_{(010)}=f\left(y_{2}\right) \in\{-1,1\}$.

2. Assume $\tilde{\chi}_{1}, \tilde{\chi}_{2}, \tilde{\chi}_{3}$ to be as in Definition 1 but now $\tilde{\chi}_{i}: \mathbb{T}^{2} \rightarrow\{-1,1\}, \mathbb{T}^{2}:=$ $\mathbb{R}^{2} / \mathbb{Z}^{2}$ and suppose that $e$ one-periodic in any coordinate direction and

$$
E_{\text {elast }}=\int_{\mathbb{T}^{2}}\left|e-\left(\begin{array}{ccc}
d_{1} & \tilde{\chi}_{3} & \tilde{\chi}_{2} \\
\tilde{\chi}_{3} & d_{2} & \tilde{\chi}_{1} \\
\tilde{\chi}_{2} & \tilde{\chi}_{1} & d_{3}
\end{array}\right)\right|^{2} d x, e=e\left(y_{1}, y_{2}\right) .
$$

Further assume that $\theta_{i}:=\left\langle\chi_{i}\right\rangle:=\int_{\mathbb{T}^{2}} \chi_{i} d x$ and that the first case in (1) holds (i.e. $\left.\tilde{\chi}_{3} \sim f_{(100)}\right)$.

Then

$$
\left|\theta_{1}\left(\theta_{2}+\theta_{4}\right)-\theta_{4}\left(\theta_{1}+\theta_{3}\right)\right| \lesssim E^{\frac{1}{4}}
$$

3. Let the same assumptions as in (2) be satisfied. Suppose $\Phi(s, t)=\left(s,-F_{(100)}(s)+\right.$ t) with $F_{(100)}^{\prime}(s)=f_{(100)}(s)$ a.e., $F_{(100)}(0)=0$.

Then there exists $g: \Phi^{-1}\left(\left[-\frac{1}{2}, \frac{1}{2}\right]^{2}\right) \rightarrow \mathbb{R},(s, t) \mapsto g(t)$, such that

$$
\left\|\tilde{\chi}_{1} \circ \Phi-g\right\|_{L^{2}\left(\Phi^{-1}\left(\left[-\frac{1}{2}, \frac{1}{2}\right]^{2}\right)\right)}^{2} \lesssim \eta^{-\frac{2}{9}} E^{\frac{5}{6}}
$$

and

$$
\left\|\tilde{\chi}_{2} \circ \Phi-\left(f_{(100)} \circ \Phi\right) g\right\|_{L^{2}\left(\Phi^{-1}\left(\left[-\frac{1}{2}, \frac{1}{2}\right]^{2}\right)\right)} \lesssim \max \left\{\eta^{-\frac{1}{9}} E^{\frac{5}{12}}, E^{\frac{1}{4}}\right\}
$$

Due to symmetry, similar results hold in the case $e=e\left(y_{1}, y_{3}\right)$ or $e=e\left(y_{2}, y_{3}\right)$. 
In this context the notation $A \lesssim B$ is used to denote that there exists a universal constant $C>0$ such that $A \leq C B$.

As rigidity estimates always imply a lower bound on the scaling of incompatible microstructures (i.e. structures which do not display the right distribution of volume fractions), and as the rigidity estimate can be complemented by an upper bound construction for incompatible microstructures, it is possible to prove the optimal scaling of incompatible microstructures. We obtain

Proposition 1. Let $\eta \ll 1$. Then there exits a family of strains $e_{\eta}$ with

$$
\begin{aligned}
\left|\theta_{1}^{\eta}\left(\theta_{2}^{\eta}+\theta_{4}^{\eta}\right)-\theta_{4}^{\eta}\left(\theta_{1}^{\eta}+\theta_{3}^{\eta}\right)\right| & \geq \frac{1}{5}, \\
\left|\theta_{1}^{\eta}\left(\theta_{2}^{\eta}+\theta_{4}^{\eta}\right)-\theta_{2}^{\eta}\left(\theta_{1}^{\eta}+\theta_{3}^{\eta}\right)\right| & \geq \frac{1}{5} .
\end{aligned}
$$

such that

$$
E_{\eta}\left(e_{\eta}, \chi^{\eta}\right) \leq c
$$

for $c>0$ independent of $\eta$. In particular, for such strains it holds

$$
\frac{1}{c} \leq \min E_{\eta}\left(e_{\eta}, \chi^{\eta}\right) \leq c
$$

for $c>0$ independent of $\eta$.

\section{The Proofs}

\subsection{Rigidity of the Outer Structure}

In this section we prove the (strong) rigidity of the outer structure of the patterns. We proceed in four major steps: Firstly, we apply the compatibility conditions for strains to obtain weak control of $\tilde{\chi}_{3}$ (c.f. Lemma 1). To be more precise, we prove control of certain second order derivatives of $\tilde{\chi}_{3}$ in $H^{-2}$. Although these expressions display the right scaling, the existence of incompatible microstructures suggests that the weak control cannot immediately be converted into strong $L^{2}$-control. For that purpose we therefore follow the strategy paved in 3 : In a second step we pass to finite differences so as to obtain weak $H^{-1}$-control of these (c.f. Lemma 2). In the decisive step we then interpolate between $B V$ and $H^{-1}$ to obtain $L^{2}$-control of $\tilde{\chi}_{3}$ (c.f. Lemma 3). Finally, we conclude as in the stress-free setting (c.f. 13]) and use a wave argument and the two-valuedness of $\tilde{\chi}_{3}$ (c.f. Proposition 21).

We begin with the weak $H^{-2}$-control: 
Lemma 1. There exist functions $\rho_{11}, \rho_{12}, \rho_{22}: B_{1}(0) \rightarrow \mathbb{R}$ such that

$$
\partial_{1} \partial_{2} \tilde{\chi}_{3}=\partial_{11} \rho_{11}+\partial_{1} \partial_{2} \rho_{12}+\partial_{22} \rho_{22} \text { in } \mathcal{D}^{\prime}
$$

and

$$
\int_{B_{1}} \rho_{11}^{2}+\rho_{12}^{2}+\rho_{22}^{2} d x \lesssim E_{\text {elast }}
$$

Proof of Lemma 1. As $e$ is a strain the compatibility conditions must be satisfied, in particular:

$$
\partial_{11}^{2} e_{22}-2 \partial_{1} \partial_{2} e_{12}+\partial_{22}^{2} e_{11}=0 .
$$

This can be exploited to derive the identity

$$
2 \partial_{1} \partial_{2} \tilde{\chi}_{3}=\partial_{11}\left(e_{22}-d_{2}\right)+2 \partial_{1} \partial_{2}\left(\tilde{\chi}_{3}-e_{12}\right)+\partial_{22}\left(e_{11}-d_{1}\right),
$$

where $d_{1}, d_{2}$ are the (constant) diagonal entries of the tensors describing the cubicto-orthorhombic phase transition. Setting

$$
\begin{aligned}
\rho_{11} & :=\frac{e_{22}-d_{2}}{2}, \\
\rho_{12} & :=\tilde{\chi}_{3}-e_{12}, \\
\rho_{22} & :=\frac{e_{11}-d_{1}}{2},
\end{aligned}
$$

and noticing that these functions are some of the components of the elastic energy the claim follows.

In order to apply the interpolation inequality we have to argue via finite differences. Hence, for $v \in \mathbb{R}^{n}, h \in \mathbb{R}$ we set

$$
\partial_{v}^{h} f(x):=f(x+h v)-f(x) .
$$

Lemma 2. There exist functions $j_{11}, j_{22}, j: B_{1}(0) \rightarrow \mathbb{R}$ such that

$$
\begin{gathered}
\partial_{1}^{h_{1}} \partial_{2}^{h_{2}} \tilde{\chi}_{3}=\partial_{1} j_{11}+\partial_{2} j_{22}+j \text { in } \mathcal{D}^{\prime}, \\
\int_{B_{1}(0)} j_{11}^{2}+j_{22}^{2}+j^{2} d x \lesssim E_{\text {elast }} .
\end{gathered}
$$

Proof of Lemma 国. The claim of the lemma follows from an integration of the identity derived in Lemma 1, We have

$$
\begin{aligned}
\partial_{1} \partial_{2} \tilde{\chi}_{3} & =\partial_{11} \rho_{11}+\partial_{1} \partial_{2} \rho_{12}+\partial_{22} \rho_{22} \\
\Leftrightarrow \partial_{1} \partial_{2}\left(\tilde{\chi}_{3}-\rho_{12}\right) & =\partial_{11} \rho_{11}+\partial_{22} \rho_{22} .
\end{aligned}
$$


Evaluating this expression at $y=x+h_{1} e_{1}+h_{2} e_{2}$ and reinterpreting the derivatives, we obtain

$$
\begin{aligned}
& \frac{d}{d h_{1}} \frac{d}{d h_{2}}\left(\tilde{\chi}_{3}-\rho_{12}\right)\left(x+h_{1} e_{1}+h_{2} e_{2}\right) \\
& =\frac{d}{d h_{1}} \partial_{1} \rho_{11}\left(x+h_{1} e_{1}+h_{2} e_{2}\right)+\frac{d}{d h_{2}} \partial_{2} \rho_{22}\left(x+h_{1} e_{1}+h_{2} e_{2}\right) .
\end{aligned}
$$

This can be integrated to arrive at

$$
\begin{aligned}
\partial_{1}^{h_{1}} & \partial_{2}^{h_{2}}\left(\tilde{\chi}_{3}-\rho_{12}\right)(x)=\int_{0}^{h_{2}} \int_{0}^{h_{1}} \frac{d}{d h_{1}^{\prime}} \frac{d}{d h_{2}^{\prime}}\left(\tilde{\chi}_{3}-\rho_{12}\right)\left(x+h_{1}^{\prime} e_{1}+h_{2}^{\prime} e_{2}\right) d h_{1}^{\prime} d h_{2}^{\prime} \\
= & \int_{0}^{h_{2}} \int_{0}^{h_{1}} \frac{d}{d h_{1}^{\prime}} \partial_{1} \rho_{11}\left(x+h_{1}^{\prime} e_{1}+h_{2}^{\prime} e_{2}\right) d h_{1}^{\prime} d h_{2}^{\prime} \\
& +\int_{0}^{h_{2}} \int_{0}^{h_{1}} \frac{d}{d h_{2}^{\prime}} \partial_{2} \rho_{22}\left(x+h_{1}^{\prime} e_{1}+h_{2}^{\prime} e_{2}\right) d h_{1}^{\prime} d h_{2}^{\prime} \\
= & \partial_{1} \int_{0}^{h_{2}} \rho_{11}\left(x+h_{1} e_{1}+h_{2}^{\prime} e_{2}\right) d h_{2}^{\prime}-\partial_{1} \int_{0}^{h_{2}} \rho_{11}\left(x+h_{2}^{\prime} e_{2}\right) d h_{2}^{\prime} \\
& +\partial_{2} \int_{0}^{h_{1}} \rho_{22}\left(x+h_{1}^{\prime} e_{1}+h_{2} e_{2}\right) d h_{1}^{\prime}-\partial_{2} \int_{0}^{h_{1}} \rho_{22}\left(x+h_{1}^{\prime} e_{1}\right) d h_{1}^{\prime} .
\end{aligned}
$$

Hence, the statement of the lemma follows with the functions

$$
\begin{aligned}
j_{11} & :=\partial_{1} \int_{0}^{h_{2}} \rho_{11}\left(x+h_{1} e_{1}+h_{2}^{\prime} e_{2}\right) d h_{2}^{\prime}-\partial_{1} \int_{0}^{h_{2}} \rho_{11}\left(x+h_{2}^{\prime} e_{2}\right) d h_{2}^{\prime}, \\
j_{22} & :=\partial_{2} \int_{0}^{h_{1}} \rho_{22}\left(x+h_{1}^{\prime} e_{1}+h_{2} e_{2}\right) d h_{1}^{\prime}-\partial_{2} \int_{0}^{h_{1}} \rho_{22}\left(x+h_{1}^{\prime} e_{1}\right) d h_{1}^{\prime}, \\
j & :=\partial_{1}^{h_{1}} \partial_{2}^{h_{2}} \rho_{12} .
\end{aligned}
$$

In the following lemma the transition from weak to strong norms is achieved via a well-known interpolation estimate, c.f. [3].

Lemma 3. Let $\eta \leq 1$. There exists a universal radius $r>0$ such that

$$
\sup _{\left|h_{1}\right|,\left|h_{2}\right| \leq r} \int_{B_{r}}\left|\partial_{1}^{h_{1}} \partial_{2}^{h_{2}} \tilde{\chi}_{3}\right| d x \lesssim \eta^{-\frac{2}{3}} E_{\text {elast }}+\eta^{\frac{1}{3}} E_{\text {surf }} .
$$


Proof. We reason via the estimate

$$
\int_{B_{r}} f^{2} d x \lesssim \eta^{\frac{1}{3}} \int_{B_{1}}|\nabla f| d x \sup |f|+\left.\left.\eta^{-\frac{2}{3}} \int_{B_{1}}|| \nabla\right|^{-1} f\right|^{2} d x
$$

applied to $\partial_{1}^{h_{1}} \partial_{2}^{h_{2}} \tilde{\chi}_{3}$. Using the discreteness of the values of $\tilde{\chi}_{3}$, we notice that the $L^{2}$-norm is equivalent to the $L^{1}$-norm and that the $L^{\infty}$-norm is bounded by a uniform constant. Thus, we conclude

$$
\int_{B_{r}}\left|\partial_{1}^{h_{1}} \partial_{2}^{h_{2}} \tilde{\chi}_{3}\right| d x \lesssim \eta^{\frac{1}{3}} \int_{B_{1}}\left|\nabla \partial_{1}^{h_{1}} \partial_{2}^{h_{2}} \tilde{\chi}_{3}\right| d x+\eta^{-\frac{2}{3}} \int_{B_{1}}\left(|j|^{2}+j_{0}^{2}\right) d x
$$

where $j=\left(\begin{array}{l}j_{1} \\ j_{2}\end{array}\right)$ and $j_{0}$ are given by Lemma 2

Having established strong control, we can mimic the wave argument from the stressfree case. To simplify notation we use the following convention:

Definition 2. Let $\{a, b\} \subset \mathbb{R}^{2}$ be a basis with dual basis given by $\left\{a^{*}, b^{*}\right\}$. For $f$ : $\mathbb{R} \rightarrow \mathbb{R}$ define the following notation $f_{a^{*}}(x):=f\left(a^{*} \cdot x\right)$.

Lemma 4. Let $\{a, b\} \subset \mathbb{R}^{2}$ be a basis. For all functions $f: B_{2}(0) \subset \mathbb{R}^{2} \rightarrow \mathbb{R}$ there exist $r>0$ and functions $g_{a^{*}}, g_{b^{*}}$ such that

$$
\int_{B_{r}}\left|f-g_{a^{*}}-g_{b^{*}}\right| d x \leq C(a, b, r) \sup _{\left|h_{a}\right|,\left|h_{b}\right| \leq 1} \int_{B_{1}}\left|\partial_{a}^{h_{a}} \partial_{b}^{h_{b}} f\right| d x .
$$

Proof of Lemma 4. As $a, b$ form a basis, we can without loss of generality assume $a=e_{1}, b=e_{2}$. Furthermore, we can replace the balls by cubes. This yields the 
following estimate

$$
\begin{aligned}
\sup _{\left|h_{1}\right|,\left|h_{2}\right| \leq 1} \int_{(-1,1)^{2}}\left|\partial_{1}^{h_{1}} \partial_{2}^{h_{2}} f\right| d x \\
\quad=\sup _{\left|h_{1}\right|,\left|h_{2}\right| \leq 1} \int_{(-1,1)^{2}}\left|f\left(x_{1}+h_{1}, x_{2}+h_{2}\right)-f\left(x_{1}+h_{1}, x_{2}\right)-f\left(x_{1}, x_{2}+h_{2}\right)+f\left(x_{1}, x_{2}\right)\right| d x \\
\quad \geq \frac{1}{4} \int_{(-1,1)^{2}} \int_{(-1,1)^{2}}\left|f\left(x_{1}+h_{1}, x_{2}+h_{2}\right)-f\left(x_{1}, x_{2}+h_{2}\right)-f\left(x_{1}+h_{1}, x_{2}\right)+f\left(x_{1}, x_{2}\right)\right| d x d h \\
\quad \geq \frac{1}{4} \int_{\left(-\frac{1}{2}, \frac{1}{2}\right)^{2}} \int_{\left(-\frac{1}{2}, \frac{1}{2}\right)^{2}}\left|f\left(y_{1}, y_{2}\right)-f\left(x_{1}, y_{2}\right)-f\left(y_{1}, x_{2}\right)+f\left(x_{1}, x_{2}\right)\right| d x d y \\
\quad \geq \frac{1}{4} \int_{\left(-\frac{1}{2}, \frac{1}{2}\right)^{2}}\left|\int_{\left(-\frac{1}{2}, \frac{1}{2}\right)^{2}} f\left(x_{1}, x_{2}\right)-f\left(x_{1}, y_{2}\right)-f\left(y_{1}, x_{2}\right)+f\left(y_{1}, y_{2}\right) d y\right| d x \\
\quad=\frac{1}{4} \int_{\left(-\frac{1}{2}, \frac{1}{2}\right)^{2}}\left|f\left(x_{1}, x_{2}\right)-\int_{-\frac{1}{2}}^{\frac{1}{2}} f\left(x_{1}, y_{2}\right) d y_{2}-\int_{-\frac{1}{2}}^{\frac{1}{2}} f\left(y_{1}, x_{2}\right) d y_{1}-\int_{\frac{1}{2}}^{\frac{1}{2}} \int_{\frac{1}{2}}^{\frac{1}{2}} f\left(y_{1}, y_{2}\right) d y_{1} d y_{2}\right| d x
\end{aligned}
$$

where $(*)$ is a consequence of Jensen's inequality.

Thus, the statement holds with the functions

$$
\begin{aligned}
& g_{(100)}\left(x_{1}\right)=\int_{-\frac{1}{2}}^{\frac{1}{2}} f\left(x_{1}, y_{2}\right) d y_{2}+\frac{1}{2} \int_{\frac{1}{2}}^{\frac{1}{2}} \int_{\frac{1}{2}}^{\frac{1}{2}} f\left(y_{1}, y_{2}\right) d y_{1} d y_{2}, \\
& g_{(010)}\left(x_{2}\right)=\int_{-\frac{1}{2}}^{\frac{1}{2}} f\left(y_{1}, x_{2}\right) d y_{1}+\frac{1}{2} \int_{\frac{1}{2}}^{\frac{1}{2}} \int_{\frac{1}{2}}^{\frac{1}{2}} f\left(y_{1}, y_{2}\right) d y_{1} d y_{2} .
\end{aligned}
$$

Proposition 2. Let $\tilde{\chi}_{3}, E$ be as in Definition 1 . Then there exist a universal radius $r>0$ and functions $f_{(100)}, f_{(010)} \in\{-1,1\}$ with the following properties:

$$
\int_{B_{r}}\left|\tilde{\chi}_{3}-f_{(100)}\right| d x \lesssim E^{\frac{1}{2}} \vee \int_{B_{r}}\left|\tilde{\chi}_{3}-f_{(010)}\right| d x \lesssim E^{\frac{1}{2}}
$$

Proof of Proposition 2. We divide the proof into several steps:

Step 1: Application of Lemma 3 and Lemma 4.

As by assumption $\tilde{\chi}_{3}$ only depends on two variables, Lemma 4 can be applied in 
combination with Lemma 3. This yields the existence of a radius $r>0$ and of functions $g_{(100)}, g_{(010)}: B_{r} \subset \mathbb{R}^{2} \rightarrow \mathbb{R}$ such that

$$
\int_{B_{r}}\left|\tilde{\chi}_{3}-g_{(100)}-g_{(010)}\right| d x \lesssim E .
$$

Step 2: There exist functions $\tilde{g}_{(100)}, \tilde{g}_{(010)}: B_{1}(0) \subset \mathbb{R}^{2} \rightarrow \mathbb{R}$ such that

$$
\begin{gathered}
\tilde{g}_{(100)} \in\left\{a_{1}-1, a_{1}+1\right\}, \tilde{g}_{(010)} \in\left\{a_{2}-1, a_{2}+1\right\}, a_{1}, a_{2} \in \mathbb{R}, \\
\int_{B_{r}}\left|\tilde{g}_{(100)}-g_{(100)}\right| d x \lesssim E, \int_{B_{r}}\left|\tilde{g}_{(010)}-g_{(010)}\right| d x \lesssim E .
\end{gathered}
$$

It suffices to prove the statement for $\tilde{g}_{(100)}$. Since for any $L^{\infty}$-function, $f$, we find $x_{2}^{*} \in(-1,1)$ such that the evaluation of $f$ at $x_{2}^{*}$ is less than the mean value of $f$ in $x_{2} \in(-1,1)$, we conclude

$$
\begin{aligned}
& \exists x_{2}^{*} \in(-r, r): \\
& \int_{(-r, r)}\left|\tilde{\chi}_{3}\left(x_{1}, x_{2}^{*}\right)-g_{(100)}\left(x_{1}\right)-g_{(010)}\left(x_{2}^{*}\right)\right| d x_{1} \\
& \leq \frac{1}{2 r} \int_{(-r, r)^{2}}\left|\tilde{\chi}_{3}\left(x_{1}, x_{2}\right)-g_{(100)}\left(x_{1}\right)-g_{(010)}\left(x_{2}\right)\right| d x .
\end{aligned}
$$

Setting

$$
\tilde{g}_{(100)}\left(x_{1}\right):=\tilde{\chi}_{3}\left(x_{1}, x_{2}^{*}\right)-g_{(010)}\left(x_{2}^{*}\right),
$$

(3) and (4) follow immediately.

An application of the triangle inequality and of step 2 implies that it suffices to show Step 3: For $\tilde{g}_{(100)}, \tilde{g}_{(010)}$ we find $a \in \mathbb{R}$ such that

$$
\begin{aligned}
& \min \left\{\int_{B_{r}}\left|\tilde{g}_{(100)}-a\right| d x, \int_{B_{r}}\left|\tilde{g}_{(010)}-a\right| d x\right\} \\
& \lesssim\left(\int_{B_{r}}\left|\tilde{\chi}_{3}-\tilde{g}_{(100)}-\tilde{g}_{(010)}\right| d x\right)^{\frac{1}{2}} \lesssim E^{\frac{1}{2}} .
\end{aligned}
$$

Let

$$
\begin{aligned}
\lambda_{1} & :=\mathcal{L}^{1}\left(\left\{x_{1} \in(-r, r) ; \tilde{g}_{(100)}\left(x_{1}\right)=a_{1}-1\right\}\right), \\
\lambda_{2} & :=\mathcal{L}^{1}\left(\left\{x_{2} \in(-r, r) ; \tilde{g}_{(010)}\left(x_{2}\right)=a_{2}-1\right\}\right), \\
\epsilon & :=\int_{(-r, r)^{2}}\left|\tilde{\chi}_{3}-\tilde{g}_{(100)}-\tilde{g}_{(010)}\right| d x .
\end{aligned}
$$


We can estimate

$$
\begin{aligned}
\epsilon \geq & \lambda_{1} \lambda_{2} \operatorname{dist}\left(a_{1}+a_{2}-2,\{-1,1\}\right) \\
& +\left(2 r-\lambda_{1}\right)\left(2 r-\lambda_{2}\right) \operatorname{dist}\left(a_{1}+a_{2}+2,\{-1,1\}\right) .
\end{aligned}
$$

Moreover, we must have that either $\operatorname{dist}\left(a_{1}+a_{2}-2,\{-1,1\}\right) \geq \frac{1}{2}$ or $\operatorname{dist}\left(a_{1}+a_{2}+2,\{-1,1\}\right) \geq \frac{1}{2}$. Otherwise, the inequality $\operatorname{dist}\left(a_{1}+a_{2}-2,\{-1,1\}\right)<\frac{1}{2}$ would imply that either

$$
a_{1}+a_{2} \in\left(\frac{1}{2}, \frac{3}{2}\right) \text { or } a_{1}+a_{2} \in\left(\frac{5}{2}, \frac{7}{2}\right) .
$$

holds. In the first case, however, this would yield

$$
a_{1}+a_{2}+2 \in\left(\frac{5}{2}, \frac{7}{2}\right)
$$

In the second case, this would result in

$$
a_{1}+a_{2}+2 \in\left(\frac{9}{2}, \frac{11}{2}\right) .
$$

Both statements contradict the assumption $\operatorname{dist}\left(a_{1}+a_{2}+2,\{-1,1\}\right)<\frac{1}{2}$. Therefore, we can w.l.o.g. assume $\operatorname{dist}\left(a_{1}+a_{2}-2,\{-1,1\}\right) \geq \frac{1}{2}$. This results in:

$$
\begin{aligned}
& 2 \epsilon \geq \lambda_{1} \lambda_{2} \\
& \Rightarrow \lambda_{1} \leq \sqrt{2} \sqrt{\epsilon} \vee \lambda_{2} \leq \sqrt{2} \sqrt{\epsilon} \\
& \Rightarrow \int_{-r}^{r}\left|\tilde{g}_{(100)}-\left(a_{1}+1\right)\right| d x_{1}=2 \lambda_{1} \leq 2 \sqrt{2 \epsilon} \lesssim E^{\frac{1}{2}} \\
& \vee \int_{-r}^{r}\left|\tilde{g}_{(010)}-\left(a_{2}+1\right)\right| d x_{2}=2 \lambda_{2} \leq 2 \sqrt{2 \epsilon} \lesssim E^{\frac{1}{2}} .
\end{aligned}
$$

An analogous argument works in case $\operatorname{dist}\left(a_{1}+a_{2}+2,\{-1,1\}\right) \geq \frac{1}{2}$.

Step 4: Conclusion.

Without loss of generality, we may suppose that the second alternative of step 2 holds, i.e. there exists $g_{(100)}:(-r, r) \rightarrow \mathbb{R}$ such that

$$
\int_{(-r, r)^{2}}\left|\tilde{\chi}_{3}\left(x_{1}, x_{2}\right)-g_{(100)}\left(x_{1}\right)\right| d x_{1} d x_{2} \lesssim E^{\frac{1}{2}} .
$$

Since $\left|g_{(100)}\right| \lesssim 1$ this leads to

$$
\int_{(-r, r)^{2}}\left|\tilde{\chi}_{3}\left(x_{1}, x_{2}\right)-g_{(100)}\left(x_{1}\right)\right|^{2} d x_{1} d x_{2} \lesssim E^{\frac{1}{2}} .
$$


As the $L^{2}$-projection on the space of constants is given by the mean value of the respective function, this implies

$$
\int_{(-r, r)^{2}}\left|\tilde{\chi}_{3}\left(x_{1}, x_{2}\right)-\frac{1}{2 r} \int_{-r}^{r} \tilde{\chi}_{3}\left(x_{1}, x_{2}^{\prime}\right) d x_{2}^{\prime}\right|^{2} d x_{1} d x_{2} \lesssim E^{\frac{1}{2}} .
$$

Defining

$$
\tilde{\chi}_{3}^{*}\left(x_{1}\right):= \begin{cases}1 ; & \frac{1}{2 r} \int_{-r}^{r} \tilde{\chi}_{3}\left(x_{1}, x_{2}\right) d x_{2} \geq 0 \\ -1 ; & \text { else }\end{cases}
$$

and remarking $\left|\tilde{\chi}_{3}\left(x_{1}, x_{2}\right)-\frac{1}{2 r} \int_{-r}^{r} \tilde{\chi}_{3}\left(x_{1}, x_{2}\right) d x_{2}\right| \geq 1$ on $\left\{\tilde{\chi}_{3} \neq \tilde{\chi}_{3}^{*}\right\}$, we note

$$
\begin{aligned}
E^{\frac{1}{2}} \gtrsim & \int_{\left\{\tilde{\chi}_{3} \neq \tilde{\chi}_{3}^{*}\right\} \cap(-r, r)^{2}}\left|\tilde{\chi}_{3}\left(x_{1}, x_{2}\right)-\frac{1}{2 r} \int_{-r}^{r} \tilde{\chi}_{3}\left(x_{1}, x_{2}^{\prime}\right) d x_{2}^{\prime}\right|^{2} d x_{1} d x_{2} \\
& \geq \mathcal{L}^{2}\left(\left\{\tilde{\chi}_{3} \neq \tilde{\chi}_{3}^{*}\right\}\right) .
\end{aligned}
$$

Hence, we obtain

$$
\begin{aligned}
& \int_{(-r, r)^{2}}\left|\tilde{\chi}_{3}-\tilde{\chi}_{3}^{*}\right|^{2} d x_{1} d x_{2}=\int_{\left\{\tilde{\chi}_{3} \neq \tilde{\chi}_{3}^{*}\right\} \cap(-r, r)^{2}}\left|\tilde{\chi}_{3}\left(x_{1}, x_{2}\right)-\tilde{\chi}_{3}^{*}\left(x_{1}\right)\right|^{2} d x_{1} d x_{2} \\
& \leq 4 \mathcal{L}^{2}\left(\left\{\tilde{\chi}_{3} \neq \tilde{\chi}_{3}^{*}\right\} \cap(-r, r)^{2}\right) \lesssim E^{\frac{1}{2}} .
\end{aligned}
$$

Setting $f_{(100)}:=\tilde{\chi}_{3}^{*}$ and noticing that the $L^{1}$-estimate follows from the $L^{2}$-estimate as a consequence of the discreteness of $\tilde{\chi}_{3}-\tilde{\chi}_{3}^{*}$, the claim follows.

\subsection{Proof of the Second Result}

In this section we consider the inner structure of the patterns. To avoid technical difficulties we work in a periodic setting. However, as the argument for the inner structure is of local nature in the stress-free case, [13, we believe that this condition can be removed and replaced with a purely local reasoning.

Again, we follow the ideas of the stress-free setting (c.f. [13]): Using the compatibility conditions, we begin with proving weak control (Lemma 5i). Via Helmholtz decomposition, we obtain that $\left(\begin{array}{c}\tilde{\chi}_{2} \\ \tilde{\chi}_{1}\end{array}\right)$ is close to a gradient field (Lemma 6). Using the method of characteristics, we argue that this gradient field is $H^{-1}$-close to a function of a single variable (Proposition 3). Last but not least, this can be translated into a statement on the volume fractions of the modified characteristic functions (Proposition 4).

We recall the setting: 
Definition 3. Let $\mathbb{T}^{d}:=\mathbb{R}^{d} / \mathbb{Z}^{d}$ and $f: \mathbb{T}^{d} \rightarrow \mathbb{R}, f \in L^{1}\left(\mathbb{T}^{d}\right)$. Define

$$
\begin{aligned}
& \mathcal{F} f(k):=\int_{\mathbb{T}^{d}} f(x) e^{-2 \pi i k \cdot x} d x, k \in \mathbb{Z}^{d}, \\
& \int_{\mathbb{Z}^{d}} h(k) d k:=\sum_{k \in \mathbb{Z}^{d}} h(k) .
\end{aligned}
$$

Let $f: \mathbb{T}^{d} \rightarrow \mathbb{R}$ be measurable. Set

$$
\begin{aligned}
& \|f\|_{H^{-s}\left(\mathbb{T}^{d}\right)}^{2}:=\int_{\mathbb{Z}^{d}} \frac{|\mathcal{F} f|^{2}}{|k|^{2 s}} d k, \\
& f \in H^{-s}\left(\mathbb{T}^{d}\right) \Leftrightarrow\|f\|_{H^{-s}\left(\mathbb{T}^{d}\right)}^{2}<\infty, \\
& \|f\|_{H_{\text {full }}^{-1}\left(\mathbb{T}^{d}\right)}^{2}:=\int_{\mathbb{Z}^{d}} \frac{1}{1+|k|^{2}}|\mathcal{F} f|^{2} d k, \\
& f \in H_{\text {full }}^{-1}\left(\mathbb{T}^{d}\right) \Leftrightarrow\|f\|_{H_{\text {full }}^{-1}\left(\mathbb{T}^{d}\right)}^{2}<\infty .
\end{aligned}
$$

Let $f: \mathbb{R}^{d} \rightarrow \mathbb{R}$ be measurable, $M \subset \mathbb{R}^{d}$ Borel, we define

$$
\langle f\rangle_{M}:=\frac{1}{\mathcal{L}^{d}(M)} \int_{M} f(y) d y .
$$

In the sequel we use the convention

$$
\begin{aligned}
& \chi_{1}, \chi_{2}, \chi_{3}, \chi_{4}: \mathbb{T}^{2} \rightarrow \mathbb{R}, \\
& \tilde{\chi}_{1}, \tilde{\chi}_{2}, \tilde{\chi}_{3}: \mathbb{T}^{2} \rightarrow \mathbb{R} \\
& E_{\text {elast }}=\int_{\left[-\frac{1}{2}, \frac{1}{2}\right]^{2}}\left|e-\left(\begin{array}{ccc}
d_{1} & \tilde{\chi}_{3} & \tilde{\chi}_{2} \\
\tilde{\chi}_{3} & d_{2} & \tilde{\chi}_{1} \\
\tilde{\chi}_{2} & \tilde{\chi}_{1} & d_{3}
\end{array}\right)\right|^{2} d y, \\
& e=e\left(y_{1}, y_{2}\right), e: \mathbb{T}^{2} \rightarrow \operatorname{Sym}(3, \mathbb{R}) .
\end{aligned}
$$

Lemma 5. For configurations in the $y_{1}, y_{2}$-plane $\left(e=e\left(y_{1}, y_{2}\right)\right)$ we have

$$
\begin{aligned}
& \partial_{1}\left(\partial_{1} \tilde{\chi}_{1}-\partial_{2} \tilde{\chi}_{2}\right)=\partial_{1} \partial_{1} \phi_{11}+\partial_{1} \partial_{2} \phi_{12}, \\
& \partial_{2}\left(\partial_{1} \tilde{\chi}_{1}-\partial_{2} \tilde{\chi}_{2}\right)=\partial_{1} \partial_{2} \rho_{12}+\partial_{2} \partial_{2} \rho_{22}
\end{aligned}
$$

and

$$
\begin{gathered}
\int_{\left[-\frac{1}{2}, \frac{1}{2}\right]^{2}} \rho_{12}^{2}+\rho_{22}^{2} d x \lesssim E_{\text {elast }} \\
\int_{\left[-\frac{1}{2}, \frac{1}{2}\right]^{2}} \phi_{11}^{2}+\phi_{12}^{2} d x \lesssim E_{\text {elast }} .
\end{gathered}
$$


In other words, for the $\left[-\frac{1}{2}, \frac{1}{2}\right]^{2}$-periodic characteristic functions $\tilde{\chi}_{2}$, $\tilde{\chi}_{1}$, this is equivalent to $\mathrm{H}^{-2}$-control:

$$
\left\|\nabla\left(\nabla \times\left(\begin{array}{c}
\tilde{\chi}_{2} \\
\tilde{\chi}_{1}
\end{array}\right)\right)\right\|_{H^{-2}\left(\mathbb{T}^{2}\right)}^{2} \lesssim E_{\text {elast }} .
$$

Proof of Lemma 5. As in Lemma 1 the statement is a result of the compatibility conditions for strains. We make use of the second block of the equations yielding

$$
\begin{aligned}
& 0=\partial_{1}\left(-\partial_{1} e_{23}+\partial_{2} e_{13}\right), \\
& 0=\partial_{2}\left(\partial_{1} e_{23}-\partial_{2} e_{13}\right),
\end{aligned}
$$

for configurations in the plane spanned by $y_{1}, y_{2}$. Thus, we obtain

$$
\begin{aligned}
\partial_{2}\left(\partial_{1} \tilde{\chi}_{1}-\partial_{2} \tilde{\chi}_{2}\right) & =\partial_{2}\left(\partial_{1}\left(\tilde{\chi}_{1}-e_{23}\right)-\partial_{2}\left(\tilde{\chi}_{2}-e_{13}\right)\right) \\
= & : \partial_{1} \partial_{2} \rho_{12}-\partial_{22} \rho_{22} .
\end{aligned}
$$

Again noticing that the $\rho_{i j}$ correspond to the components of the elastic energy, we obtain $L^{2}$-control:

$$
\int_{\left[-\frac{1}{2}, \frac{1}{2}\right]^{2}} \rho_{12}^{2}+\rho_{22}^{2} d x \lesssim E_{\text {elast }}
$$

The second statement follows in the same way.

Lemma 6. Let $w: \mathbb{T}^{2} \rightarrow \mathbb{R}^{2}, w \in L^{2}\left(\mathbb{T}^{2}\right)$, then we have

$$
\|P w\|_{L^{2}\left(\mathbb{T}^{2}\right)}=\|\nabla \times w\|_{H^{-1}\left(\mathbb{T}^{2}\right)},
$$

where $P$ denotes the Leray-projection.

Proof of Lemma 6. Working in Fourier space, the Leray-projection takes the following form:

$$
\mathcal{F}(P w)=\mathcal{F} w-\frac{k \cdot \mathcal{F} w}{|k|^{2}} k=\frac{|k|^{2} \mathcal{F} w-k \cdot \mathcal{F} w}{|k|^{2}} .
$$

With the identity

$$
k \times(k \times \mathcal{F} w)=|k|^{2} \mathcal{F} w-k \cdot \mathcal{F} w,
$$

we obtain

$$
\begin{aligned}
|\mathcal{F}(P w)|^{2} & =\frac{|k \times(k \times \mathcal{F} w)|^{2}}{|k|^{4}} \\
& =\frac{|k \times \mathcal{F} w|^{2}}{|k|^{2}},
\end{aligned}
$$

which proves the claim. 
Proposition 3. Let $\tilde{\chi}_{1}, \tilde{\chi}_{2}, \tilde{\chi}_{3}, E_{\text {elast }}$ be as in Definition 3. Define $\Phi(s, t):=(s, t-$ $\left.F_{(100)}(s)\right)$ where $F_{(100)}^{\prime}(s)=f_{(100)}(s)$ a.e., $F_{(100)}(0)=0$ and where $f_{(100)}$ is the function from Proposition 2 , Then we have:

1. There exists $u:\left[-\frac{1}{2}, \frac{1}{2}\right]^{2} \rightarrow \mathbb{R}, u \in H^{1}\left(\left[-\frac{1}{2}, \frac{1}{2}\right]^{2}\right),\left[-\frac{1}{2}, \frac{1}{2}\right]^{2}$ - periodic, and there exists $g: \Phi^{-1}\left(\left[-\frac{1}{2}, \frac{1}{2}\right]^{2}\right) \subset \mathbb{R}^{2} \rightarrow \mathbb{R},(s, t) \mapsto g(t)$, one-periodic, such that

$$
\begin{aligned}
& \left\|\left(\begin{array}{c}
\tilde{\chi}_{2} \\
\tilde{\chi}_{1}
\end{array}\right)-\nabla u\right\|_{L^{2}\left(\left[-\frac{1}{2}, \frac{1}{2}\right]^{2}\right)} \lesssim E_{\text {elast }}^{\frac{1}{2}}, \\
& \|u \circ \Phi-g\|_{L^{2}\left(\Phi^{-1}\left(\left[-\frac{1}{2}, \frac{1}{2}\right]^{2}\right)\right)} \lesssim E^{\frac{1}{4}} .
\end{aligned}
$$

2. Let $\tilde{g}: \mathbb{R}^{2} \rightarrow \mathbb{R},\left(y_{1}, y_{2}\right) \mapsto \tilde{g}\left(y_{1}, y_{2}\right)$ be such that $\tilde{g}\left(y_{1}, y_{2}\right)=\left(g \circ \Phi^{-1}\right)\left(y_{1}, y_{2}\right)$ for $\left(y_{1}, y_{2}\right) \in\left[-\frac{1}{2}, \frac{1}{2}\right]^{2}$ and let $\tilde{g}$ be $\left[-\frac{1}{2}, \frac{1}{2}\right]^{2}$-periodically continued. Then we have

$$
\left\|\left(\begin{array}{c}
\tilde{\chi}_{2} \\
\tilde{\chi}_{1}
\end{array}\right)-\left(\begin{array}{c}
f_{(100)} \partial_{2} \tilde{g} \\
\partial_{2} \tilde{g}
\end{array}\right)\right\|_{H_{\text {full }}^{-1}\left[\left[-\frac{1}{2}, \frac{1}{2}\right]^{2}\right)} \lesssim E^{\frac{1}{4}} .
$$

Remark 1. 1. Let $f: \mathbb{R}^{2} \rightarrow \mathbb{R},(s, t) \mapsto f(s, t)$.

As $\operatorname{det}(D \Phi)(s, t)=1$, we have

$$
\|f\|_{L^{2}\left(\Phi^{-1}\left(\left[-\frac{1}{2}, \frac{1}{2}\right]^{2}\right)\right)}=\left\|f \circ \Phi^{-1}\right\|_{L^{2}\left(\left[-\frac{1}{2}, \frac{1}{2}\right]^{2}\right)} .
$$

2. In the periodic setting the statement of Proposition 3 amounts to

$$
\left\|\tilde{\chi}_{3}-f_{(100)}\right\|_{L^{2}\left(\left[-\frac{1}{2}, \frac{1}{2}\right]^{2}\right)} \lesssim E^{\frac{1}{4}} .
$$

Mimicking the proof of the stress-free case, we proceed in several steps: Via the compatibility conditions we prove closeness of $\left(\begin{array}{c}\tilde{\chi}_{2} \\ \tilde{\chi}_{1}\end{array}\right)$ to a gradient field $\nabla u$ for which we determine the characteristic equations. An application of Poincaré's inequality and a change of coordinates yield closeness of $u$ to a function of a single variable. Since $u$ resembles, loosely speaking, the inverse gradient of $\left(\begin{array}{c}\tilde{\chi}_{2} \\ \tilde{\chi}_{1}\end{array}\right)$, this motivates the closeness of this vector field to the crossing twin structures with respect to the $H^{-1}$-norm. Without additionally making use of the surface energy this is optimal (c.f. counterexample given in Lemma (7).

Proof. Step 1: $H^{-1}$-control.

Lemma 5 yields

$$
\left\|\nabla\left(\nabla \times\left(\begin{array}{c}
\tilde{\chi}_{2} \\
\tilde{\chi}_{1}
\end{array}\right)\right)\right\|_{H^{-2}\left(\left[-\frac{1}{2}, \frac{1}{2}\right]^{2}\right)} \lesssim E_{\text {elast }}^{\frac{1}{2}}
$$


Using the periodicity assumptions this immediately translates into

$$
\left\|\nabla \times\left(\begin{array}{c}
\tilde{\chi}_{2} \\
\tilde{\chi}_{1}
\end{array}\right)\right\|_{H^{-1}\left(\left[-\frac{1}{2}, \frac{1}{2}\right]^{2}\right)}=\left\|\nabla\left(\nabla \times\left(\begin{array}{c}
\tilde{\chi}_{2} \\
\tilde{\chi}_{1}
\end{array}\right)\right)\right\|_{H^{-2}\left(\left[-\frac{1}{2}, \frac{1}{2}\right]^{2}\right)} \lesssim E_{\text {elast }}^{\frac{1}{2}},
$$

which is the estimate we looked for.

Step 2: $L^{2}$-control.

Denoting the Leray projection with $P$ and referring to Lemma [6, we obtain

$$
\left\|P\left(\left(\begin{array}{c}
\tilde{\chi}_{2} \\
\tilde{\chi}_{1}
\end{array}\right)\right)\right\|_{L^{2}\left(\left[-\frac{1}{2}, \frac{1}{2}\right]^{2}\right)} \stackrel{\underline{\underline{5}}}{=}\left\|\nabla \times\left(\begin{array}{c}
\tilde{\chi}_{2} \\
\tilde{\chi}_{1}
\end{array}\right)\right\|_{H^{-1}\left(\left[-\frac{1}{2}, \frac{1}{2}\right]^{2}\right)} \lesssim E_{\text {elast }}^{\frac{1}{2}} .
$$

With the Helmholtz-projection on gradient fields, $Q$, this turns into

$$
\left\|\left(\begin{array}{c}
\tilde{\chi}_{2} \\
\tilde{\chi}_{1}
\end{array}\right)-Q\left(\begin{array}{c}
\tilde{\chi}_{2} \\
\tilde{\chi}_{1}
\end{array}\right)\right\|_{L^{2}\left(\left[-\frac{1}{2}, \frac{1}{2}\right]^{2}\right)} \lesssim E_{\text {elast }}^{\frac{1}{2}}
$$

and

$$
\begin{aligned}
& \left(\begin{array}{c}
\partial_{1} u \\
\partial_{2} u
\end{array}\right):=Q\left(\begin{array}{c}
\tilde{\chi}_{2} \\
\tilde{\chi}_{1}
\end{array}\right) \in L^{2}\left(\left[-\frac{1}{2}, \frac{1}{2}\right]^{2}\right), u \in H^{1}\left(\left[-\frac{1}{2}, \frac{1}{2}\right]^{2}\right), \\
& u\left[-\frac{1}{2}, \frac{1}{2}\right]^{2} \text { - periodic. }
\end{aligned}
$$

Step 3: Characteristics for u: We have

$$
\left\|\frac{d}{d s}(u \circ \Phi)\right\|_{L^{2}\left(\Phi^{-1}\left(\left[-\frac{1}{2}, \frac{1}{2}\right]^{2}\right)\right)} \lesssim E^{\frac{1}{4}} .
$$

As in the stress-free setting we exploit the structure of the strains describing the phase transition. All in all, we have the following identities and estimates at our disposal:

$$
\begin{aligned}
& \tilde{\chi}_{2}-\tilde{\chi}_{3} \tilde{\chi}_{1}=0, \\
& \left\|\tilde{\chi}_{3}-f_{(100)}\right\|_{L^{2}\left(\left[-\frac{1}{2}, \frac{1}{2}\right]^{2}\right)} \lesssim E^{\frac{1}{4}}, \\
& \left\|\left(\begin{array}{c}
\tilde{\chi}_{2} \\
\tilde{\chi}_{1}
\end{array}\right)-Q\left(\begin{array}{c}
\tilde{\chi}_{2} \\
\tilde{\chi}_{1}
\end{array}\right)\right\|_{L^{2}\left(\left[-\frac{1}{2}, \frac{1}{2}\right]^{2}\right)} \lesssim E_{\text {elast }}^{\frac{1}{2}}, \\
& \left|f_{(100)}\right|=1, \quad \eta \leq 1 .
\end{aligned}
$$


Since $\Phi(s, t)$ is a bilipschitz mapping the chain rule may be applied almost everywhere:

$$
\begin{aligned}
& \left\|\frac{d}{d s}(u \circ \Phi)\right\|_{L^{2}\left(\Phi^{-1}\left(\left[-\frac{1}{2}, \frac{1}{2}\right]^{2}\right)\right)}=\left\|\partial_{1} u \circ \Phi-\left(f_{(100)} \circ \Phi\right)\left(\partial_{2} u \circ \Phi\right)\right\|_{L^{2}\left(\Phi^{-1}\left(\left[-\frac{1}{2}, \frac{1}{2}\right]^{2}\right)\right)} \\
& \stackrel{(77}{=}\left\|\partial_{1} u-f_{(100)} \partial_{2} u+f_{(100)} \tilde{\chi}_{1}-f_{(100)} \tilde{\chi}_{1}-\tilde{\chi}_{2}+\tilde{\chi}_{3} \tilde{\chi}_{1}\right\|_{L^{2}\left(\left[-\frac{1}{2}, \frac{1}{2}\right]^{2}\right)}
\end{aligned}
$$

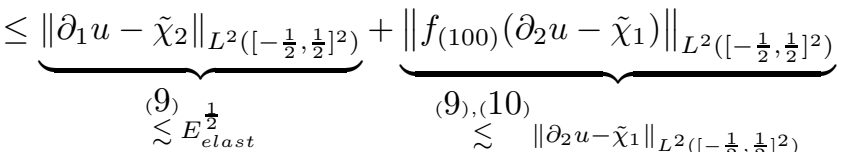

$$
\begin{aligned}
& \lesssim\left\|\partial_{2} u-\tilde{\chi}_{1}\right\|_{L^{2}\left(\left[-\frac{1}{2}, \frac{1}{2}\right]^{2}\right)} \\
& +\underbrace{\left\|\tilde{\chi}_{1}\left(f_{(100)}-\tilde{\chi}_{3}\right)\right\|_{L^{2}\left(\left[-\frac{1}{2}, \frac{1}{2}\right]^{2}\right)}}_{\leq\left\|\tilde{\chi}_{1}\right\|_{L^{\infty}}\left\|f_{(100)}-\tilde{\chi}_{3}\right\|_{L^{2}\left(\left[-\frac{1}{2}, \frac{1}{2}\right]^{2}\right)}} \stackrel{9], 8]}{\lesssim} E^{\frac{1}{4}} .
\end{aligned}
$$

Step 4: Application of Poincaré's inequality.

As $\Phi$ is bilipschitz, there exist $k \in \mathbb{N}$ and a cube $\left[-\frac{r}{2}, \frac{r}{2}\right]^{2}$, such that the inclusions $\Phi^{-1}\left(\left[-\frac{1}{2}, \frac{1}{2}\right]^{2}\right) \subset\left[-\frac{r}{2}, \frac{r}{2}\right]^{2} \subset \Phi^{-1}\left(\left[-\frac{k}{2}, \frac{k}{2}\right]^{2}\right)$ hold. Due to the $\left[-\frac{1}{2}, \frac{1}{2}\right]^{2}-$ periodicity of $u$ we have

$$
\left\|\frac{d}{d s}(u \circ \Phi)\right\|_{L^{2}\left(\Phi^{-1}\left(\left[-\frac{k}{2}, \frac{k}{2}\right]^{2}\right)\right)} \leq C k^{2}\left\|\frac{d}{d s}(u \circ \Phi)\right\|_{L^{2}\left(\Phi^{-1}\left(\left[-\frac{1}{2}, \frac{1}{2}\right]^{2}\right)\right)} \lesssim E^{\frac{1}{4}} .
$$

Applying the lemma of Poincaré we find $g:\left[-\frac{r}{2}, \frac{r}{2}\right]^{2} \subset \mathbb{R}^{2} \rightarrow \mathbb{R},(s, t) \mapsto g(t)$, oneperiodic, such that

$$
\|u \circ \Phi-g\|_{L^{2}\left(\left[-\frac{r}{2}, \frac{r}{2}\right]^{2}\right)} \lesssim\left\|\frac{d}{d s}(u \circ \Phi)\right\|_{L^{2}\left(\left[-\frac{r}{2}, \frac{r}{2}\right]^{2}\right)} .
$$

Therefore we conclude

$$
\begin{aligned}
\| u \circ \Phi & -g\left\|_{L^{2}\left(\Phi^{-1}\left(\left[-\frac{1}{2}, \frac{1}{2}\right]^{2}\right)\right)} \leq\right\| u \circ \Phi-g \|_{L^{2}\left(\left[-\frac{r}{2}, \frac{r}{2}\right]^{2}\right)} \\
& \lesssim\left\|\frac{d}{d s}(u \circ \Phi)\right\|_{L^{2}\left(\left[-\frac{r}{2}, \frac{r}{2}\right]^{2}\right)} \leq\left\|\frac{d}{d s}(u \circ \Phi)\right\|_{L^{2}\left(\Phi^{-1}\left(\left[-\frac{k}{2}, \frac{k}{2}\right]^{2}\right)\right)} \\
& \lesssim E^{\frac{1}{4}}
\end{aligned}
$$

Step 5: Proof of part 2.

We estimate:

$$
\begin{aligned}
& \left\|\left(\begin{array}{c}
\tilde{\chi}_{2} \\
\tilde{\chi}_{1}
\end{array}\right)-\left(\begin{array}{c}
f_{(100)} \partial_{2} \tilde{g} \\
\partial_{2} \tilde{g}
\end{array}\right)\right\|_{H_{\text {full }}^{-1}\left(\mathbb{T}^{2}\right)} \leq\left\|\left(\begin{array}{c}
\tilde{\chi}_{2} \\
\tilde{\chi}_{1}
\end{array}\right)-\nabla u\right\|_{H_{\text {full }}^{-1}\left(\mathbb{T}^{2}\right)} \\
& \quad+\left\|\left(\begin{array}{c}
f_{(100)} \partial_{2} \tilde{g} \\
\partial_{2} \tilde{g}
\end{array}\right)-\left(\begin{array}{c}
f_{(100)} \partial_{2} u \\
\partial_{2} u
\end{array}\right)\right\|_{H_{f u l l}^{-1}\left(\mathbb{T}^{2}\right)} \\
& +\left\|\partial_{1} u-f_{(100)} \partial_{2} u\right\|_{H_{f u l l}^{-1}\left(\mathbb{T}^{2}\right)} .
\end{aligned}
$$


Using the continuous embedding $L^{2} \hookrightarrow H_{\text {full }}^{-1}$ and recalling (9), we can deal with the first term:

$$
\left\|\left(\begin{array}{c}
\tilde{\chi}_{2} \\
\tilde{\chi}_{1}
\end{array}\right)-\nabla u\right\|_{H_{\text {full }}^{-1}\left(\mathbb{T}^{2}\right)} \lesssim\left\|\left(\begin{array}{c}
\tilde{\chi}_{2} \\
\tilde{\chi}_{1}
\end{array}\right)-\nabla u\right\|_{L^{2}\left(\mathbb{T}^{2}\right)} \lesssim E_{\text {elast }}^{\frac{1}{2}}
$$

For the third term we recall that by definition of $\Phi$ the identity

$$
\left(\partial_{1} u-f_{(100)} \partial_{2} u\right) \circ \Phi=\frac{d}{d s}(u \circ \Phi)
$$

holds. Taking into account (6) and Remark 1, we obtain:

$$
\begin{gathered}
\left\|\partial_{1} u-f_{(100)} \partial_{2} u\right\|_{H_{f u l l}^{-1}\left(\mathbb{T}^{2}\right)} \lesssim\left\|\partial_{1} u-f_{(100)} \partial_{2} u\right\|_{L^{2}\left(\mathbb{T}^{2}\right)} \\
=\left\|\frac{d}{d s}(u \circ \Phi)\right\|_{L^{2}\left(\Phi^{-1}\left(\mathbb{T}^{2}\right)\right)} \stackrel{\sqrt[6]{6}}{\lesssim} E^{\frac{1}{4}} .
\end{gathered}
$$

In order to bound the second term we use that $f_{(100)}$ only depends on $y_{1}$. Further we remark that $\partial_{2} \tilde{g}$ exists in the Sobolev sense as $g$ is one-periodic and $\tilde{g}$ is obtained from $g$ via periodizing in $y_{1}$-direction. Consequently:

$$
\begin{aligned}
\left\|\left(\begin{array}{c}
f_{(100)} \partial_{2} \tilde{g} \\
\partial_{2} \tilde{g}
\end{array}\right)-\left(\begin{array}{c}
f_{(100)} \partial_{2} u \\
\partial_{2} u
\end{array}\right)\right\|_{H_{f u l l}^{-1}\left(\mathbb{T}^{2}\right)} & =\left\|\partial_{2}\left(\left(\begin{array}{c}
f_{(100)} \tilde{g} \\
\tilde{g}
\end{array}\right)-\left(\begin{array}{c}
f_{(100)} u \\
u
\end{array}\right)\right)\right\|_{H_{f u l l}^{-1}\left(\mathbb{T}^{2}\right)} \\
& \leq\left\|\left(\begin{array}{c}
f_{(100)} \tilde{g} \\
\tilde{g}
\end{array}\right)-\left(\begin{array}{c}
f_{(100)} u \\
u
\end{array}\right)\right\|_{L^{2}\left(\mathbb{T}^{2}\right)} .
\end{aligned}
$$

Due to (10), we deduce

$$
\begin{aligned}
& \left\|\left(\begin{array}{c}
f_{(100)} \tilde{g} \\
\tilde{g}
\end{array}\right)-\left(\begin{array}{c}
f_{(100)} u \\
u
\end{array}\right)\right\|_{L^{2}\left(\mathbb{T}^{2}\right)} \leq 2\|\tilde{g}-u\|_{L^{2}\left(\mathbb{T}^{2}\right)} \\
& \quad \lesssim\left\|g \circ \Phi^{-1}-u\right\|_{L^{2}\left(\mathbb{T}^{2}\right)} \\
& \quad=\|g-u \circ \Phi\|_{L^{2}\left(\Phi^{-1}\left(\left[-\frac{1}{2}, \frac{1}{2}\right]^{2}\right)\right)} \lesssim E^{\frac{1}{4}} .
\end{aligned}
$$

Thus, we combine these estimates to conclude

$$
\left\|\left(\begin{array}{c}
\tilde{\chi}_{2} \\
\tilde{\chi}_{1}
\end{array}\right)-\left(\begin{array}{c}
f_{(100)} \partial_{2} \tilde{g} \\
\partial_{2} \tilde{g}
\end{array}\right)\right\|_{H_{\text {full }}^{-1}\left(\mathbb{T}^{2}\right)} \lesssim E^{\frac{1}{4}}
$$

In order derive a statement on the volume fractions, we have to exploit the properties of the approximating functions associated to the modified characteristic functions. Under the change of coordinates, $\Phi$, the functions $f_{(100)}$ and $\tilde{g}$ approximating $\tilde{\chi}_{3}$ and $\tilde{\chi}_{1}$ respectively are, roughly speaking, independent. The good properties of the change of coordinates preserve this. 
Proposition 4. Let $\eta \leq 1$ and assume $\tilde{\chi}_{i}, E, E_{\text {elast }}$ are as in Definition [3, suppose that $\left\|\tilde{\chi}_{3}-f_{(100)}\right\|_{L^{2}\left(\mathbb{T}^{2}\right)} \lesssim E^{\frac{1}{4}}$. Let $\Phi(s, t):=\left(s, t-F_{(100)}(s)\right), F_{(100)}^{\prime}(s)=f_{(100)}(s)$ a.e., $F_{(100)}(0)=0$. Then it holds

$$
\left|\theta_{1}\left(\theta_{2}+\theta_{4}\right)-\theta_{4}\left(\theta_{1}+\theta_{3}\right)\right| \lesssim E^{\frac{1}{4}}
$$

Proof of Proposition 4 . Step 1: Uncorrelatedness. Let $f, g: \mathbb{R}^{2} \rightarrow \mathbb{R} ;$ suppose $f, g$ to be one-periodic in $y_{2}$ and assume

$$
\begin{aligned}
& (f \circ \Phi)(s, t)=h(s), \\
& (g \circ \Phi)(s, t)=l(t) .
\end{aligned}
$$

Then we have (using the notation of Definition [3)

$$
\langle f\rangle_{\mathbb{T}^{2}}\langle g\rangle_{\mathbb{T}^{2}}=\langle f g\rangle_{\mathbb{T}^{2}}
$$

Exploiting the properties of $\Phi$ and using the periodicity of $f, g$, we obtain:

$$
\begin{aligned}
\int_{\Phi\left(\left[-\frac{1}{2}, \frac{1}{2}\right]^{2}\right)} f\left(y_{1}, y_{2}\right) d y_{1} d y_{2} & =\int_{-\frac{1}{2}-F_{(100)}\left(y_{1}\right)-\frac{1}{2}}^{\frac{1}{2}} f\left(y_{1}, y_{2}\right) d y_{2} d y_{1} \\
& =\int_{-\frac{1}{2}}^{\frac{1}{2}} \int_{-\frac{1}{2}}^{\frac{1}{2}} f\left(y_{1}, y_{2}\right) d y_{1} d y_{2} .
\end{aligned}
$$

Thus, we have

$$
\begin{aligned}
& \langle f\rangle_{\mathbb{T}^{2}}=\langle f\rangle_{\Phi\left(\left[-\frac{1}{2}, \frac{1}{2}\right]^{2}\right)}, \\
& \langle g\rangle_{\mathbb{T}^{2}}=\langle g\rangle_{\Phi\left(\left[-\frac{1}{2}, \frac{1}{2}\right]^{2}\right)}, \\
& \langle f g\rangle_{\mathbb{T}^{2}}=\langle f g\rangle_{\Phi\left(\left[-\frac{1}{2}, \frac{1}{2}\right]^{2}\right)} .
\end{aligned}
$$

An application of Fubini's theorem finally proves the claim

$$
\begin{aligned}
\langle f\rangle_{\mathbb{T}^{2}}\langle g\rangle_{\mathbb{T}^{2}} & =\langle f\rangle_{\Phi\left(\left[-\frac{1}{2}, \frac{1}{2}\right]^{2}\right)}\langle g\rangle_{\Phi\left(\left[-\frac{1}{2}, \frac{1}{2}\right]^{2}\right)}=\langle f \circ \Phi\rangle_{\left[-\frac{1}{2}, \frac{1}{2}\right]^{2}}\langle g \circ \Phi\rangle_{\left[-\frac{1}{2}, \frac{1}{2}\right]^{2}} \\
& =\left(\int_{-\frac{1}{2}}^{\frac{1}{2}} h(s) d s\right)\left(\int_{-\frac{1}{2}}^{\frac{1}{2}} l(t) d t\right)=\int_{-\frac{1}{2}}^{\frac{1}{2}} \int_{-\frac{1}{2}}^{\frac{1}{2}} h(s) l(t) d s d t \\
& =\langle(f g) \circ \Phi\rangle_{\left[-\frac{1}{2}, \frac{1}{2}\right]^{2}}=\langle f g\rangle_{\Phi\left(\left[-\frac{1}{2}, \frac{1}{2}\right]^{2}\right)}=\langle f g\rangle_{\mathbb{T}^{2}} .
\end{aligned}
$$

Step 2: Proof of the proposition.

By definition of $\tilde{\chi}_{i}$ we can rewrite the expressions $\theta_{1}\left(\theta_{2}+\theta_{4}\right), \theta_{4}\left(\theta_{1}+\theta_{3}\right)$ as combinations 
of the modified characteristic functions:

$$
\begin{aligned}
& \theta_{1}\left(\theta_{2}+\theta_{4}\right)=\left\langle\frac{1-\tilde{\chi}_{3}}{2}\right\rangle\left\langle\frac{1}{4}\left(1+\tilde{\chi}_{2}+\tilde{\chi}_{3}+\tilde{\chi}_{1}\right)\right\rangle \\
& \theta_{4}\left(\theta_{1}+\theta_{3}\right)=\left\langle 1-\frac{1-\tilde{\chi}_{3}}{2}\right\rangle\left\langle\frac{1}{4}\left(1-\tilde{\chi}_{2}-\tilde{\chi}_{3}+\tilde{\chi}_{1}\right)\right\rangle .
\end{aligned}
$$

Due to the $H_{\text {full }}^{-1}$-estimate from Proposition 3 , the mean values of $\tilde{\chi}_{2}, \tilde{\chi}_{1}$ are controlled by functions describing the crossing twin structures:

$$
\begin{aligned}
& \left(\sum_{k \in \mathbb{Z}^{2}} \frac{1}{1+|k|^{2}}\left|\left(\begin{array}{c}
\left(\mathcal{F} \tilde{\chi}_{2}\right)(k) \\
\left(\mathcal{F} \tilde{\chi}_{1}\right)(k)
\end{array}\right)-\left(\begin{array}{c}
\left(\mathcal{F} f_{(100)} \partial_{2} \tilde{g}\right)(k) \\
\mathcal{F}\left(\partial_{2} \tilde{g}\right)(k)
\end{array}\right)\right|^{2}\right)^{\frac{1}{2}} \lesssim E^{\frac{1}{4}} \\
& \Rightarrow\left|\left(\begin{array}{c}
\left(\mathcal{F} \tilde{\chi}_{2}\right)(0) \\
\left(\mathcal{F} \tilde{\chi}_{1}\right)(0)
\end{array}\right)-\left(\begin{array}{c}
\left(\mathcal{F} f_{(100)} \partial_{2} \tilde{g}\right)(0) \\
\left(\mathcal{F} \partial_{2} \tilde{g}\right)(0)
\end{array}\right)\right| \lesssim E^{\frac{1}{4}} \\
& \Leftrightarrow\left\{\begin{array}{l}
\left|\left\langle\tilde{\chi}_{2}\right\rangle-\left\langle f_{(100)} \partial_{2} \tilde{g}\right\rangle\right| \lesssim E^{\frac{1}{4}} \\
\left|\left\langle\tilde{\chi}_{1}\right\rangle-\left\langle\partial_{2} \tilde{g}\right\rangle\right| \lesssim E^{\frac{1}{4}}
\end{array}\right.
\end{aligned}
$$

As multiplicative constants are irrelevant for the scaling behavior, we can ignore the factor $\frac{1}{4}$ in the expressions (11), (12). As a consequence of (13) and (8) we can - taking into account an error of $E^{\frac{1}{4}}$ - work with the approximative quantities:

$$
\begin{aligned}
& \left\langle 1+f_{(100)}+\partial_{2} \tilde{g}+f_{(100)} \partial_{2} \tilde{g}\right\rangle\left\langle\frac{1-f_{(100)}}{2}\right\rangle \\
= & \left\langle 1-f_{(100)} \partial_{2} \tilde{g}-f_{(100)}+\partial_{2} \tilde{g}\right\rangle\left\langle 1-\frac{1-f_{(100)}}{2}\right\rangle \\
\Leftrightarrow & \left\langle 1+\partial_{2} \tilde{g}\right\rangle\left\langle 1-f_{(100)}\right\rangle=\left\langle 1-f_{(100)} \partial_{2} \tilde{g}-f_{(100)}+\partial_{2} \tilde{g}\right\rangle .
\end{aligned}
$$

Remembering $(\tilde{g} \circ \Phi)(s, t)=g(t)$ for $s \in[a, a+1]$, where $a=\frac{k}{2}, k \in \mathbb{Z}$, step 1 yields

$$
\left\langle 1+\partial_{2} \tilde{g}\right\rangle\left\langle 1-f_{(100)}\right\rangle=\left\langle\left(1+\partial_{2} \tilde{g}\right)\left(1-f_{(100)}\right)\right\rangle .
$$

\subsection{Proof of the Third Statement}

In this section we show that we can not only prove weak rigidity in the sense of having approximately the right volume fractions, but that we can also obtain rigidity in a strong norm. As we use the interpolation inequality again, we however give up a factor of $\eta^{-\frac{2}{9}}$ in the scaling behavior.

Thus, the question whether we need the BV control once more can be posed. As a counterexample (c.f. Lemma 7) at the end of the section proves, this is indeed a necessary condition. Yet, the counterexample does not imply that the use of the BV control enforces a loss in the scaling behavior. 
Proposition 5. Let $\eta \leq 1$. Assume $\tilde{\chi}_{1}, \tilde{\chi}_{2}, \tilde{\chi}_{3}: \mathbb{T}^{2} \rightarrow \mathbb{R}, E_{\text {elast }}, E$ are as in Definition 0, Let

$$
\Phi(s, t):=\left(s, t-F_{(100)}(s)\right)
$$

with $F_{(100)}^{\prime}(s):=f_{(100)}(s)$ a.e., $F_{(100)}(0)=0$.

Then there exists $g: \Phi^{-1}\left(\left[-\frac{1}{2}, \frac{1}{2}\right]^{2}\right) \rightarrow \mathbb{R},(s, t) \mapsto g(t)$ such that

$$
\left\|\tilde{\chi}_{1} \circ \Phi-g\right\|_{L^{2}\left(\Phi^{-1}\left(\left[-\frac{1}{2}, \frac{1}{2}\right]^{2}\right)\right)}^{2} \lesssim \eta^{-\frac{2}{9}} E^{\frac{5}{6}}
$$

and

$$
\left\|\tilde{\chi}_{2} \circ \Phi-\left(f_{(100)} \circ \Phi\right) g\right\|_{L^{2}\left(\Phi^{-1}\left(\left[-\frac{1}{2}, \frac{1}{2}\right]^{2}\right)\right)} \lesssim \max \left\{\eta^{-\frac{1}{9}} E^{\frac{5}{12}}, E^{\frac{1}{4}}\right\} .
$$

Proof. Step 1: We have

$$
\left\|\nabla \cdot\left(\begin{array}{c}
\tilde{\chi}_{1} \\
-f_{(100)} \tilde{\chi}_{1}
\end{array}\right)\right\|_{H^{-1}\left(\mathbb{T}^{2}\right)} \lesssim E^{\frac{1}{4}} .
$$

This is a consequence of the estimate for $\tilde{\chi}_{3}$ as well as the characteristics. We have

$$
\begin{aligned}
& \tilde{\chi}_{2}-\tilde{\chi}_{3} \tilde{\chi}_{1}=0, \\
& \left\|\tilde{\chi}_{3}-f_{(100)}\right\|_{L^{2}\left(\left[-\frac{1}{2}, \frac{1}{2}\right]^{2}\right)} \lesssim E^{\frac{1}{4}} .
\end{aligned}
$$

Combining these relations with Lemma 5, we obtain

$$
\begin{aligned}
\left\|\nabla \cdot\left(\begin{array}{cc}
\tilde{\chi}_{1} \\
-f_{(100)} \tilde{\chi}_{1}
\end{array}\right)\right\|_{H^{-1}\left(\mathbb{T}^{2}\right)} & =\left\|\nabla \cdot\left(\begin{array}{c}
\tilde{\chi}_{1} \\
-f_{(100)} \tilde{\chi}_{1}
\end{array}\right)-\nabla \cdot\left(\begin{array}{c}
\tilde{\chi}_{1} \\
-\tilde{\chi}_{3} \tilde{\chi}_{1}
\end{array}\right)\right\|_{H^{-1}\left(\mathbb{T}^{2}\right)} \\
& +\left\|\nabla \cdot\left(\begin{array}{c}
\tilde{\chi}_{1} \\
-\tilde{\chi}_{3} \tilde{\chi}_{1}
\end{array}\right)\right\|_{H^{-1}\left(\mathbb{T}^{2}\right)} \\
& \stackrel{155}{\leq}\left\|\tilde{\chi}_{1}\left(-f_{(100)}+\tilde{\chi}_{3}\right)\right\|_{L^{2}\left(\mathbb{T}^{2}\right)}+\left\|\partial_{1} \tilde{\chi}_{1}-\partial_{2} \tilde{\chi}_{2}\right\|_{H^{-1}\left(\mathbb{T}^{2}\right)} \\
& \stackrel{116}{\lesssim} E^{\frac{1}{4}}+E_{\text {elast }}^{\frac{1}{2}},
\end{aligned}
$$

as Lemma 5 states

$$
\left\|\partial_{1} \tilde{\chi}_{1}-\partial_{2} \tilde{\chi}_{2}\right\|_{H^{-1}\left(\mathbb{T}^{2}\right)}=\left\|\nabla\left(\partial_{1} \tilde{\chi}_{1}-\partial_{2} \tilde{\chi}_{2}\right)\right\|_{H^{-2}\left(\mathbb{T}^{2}\right)} \lesssim E_{\text {elast }}^{\frac{1}{2}} .
$$

Step 2: There exists $j_{\tau}, j: \Phi^{-1}\left(\left[-\frac{1}{2}, \frac{1}{2}\right]^{2}\right) \rightarrow \mathbb{R}$ such that

$$
\begin{gathered}
\partial_{s}^{h} \tilde{\chi}_{1}(\Phi(s, t))=\partial_{t} j_{\tau}(s, t)+j(s, t), \\
\int_{\Phi^{-1}\left(\left[-\frac{1}{2}, \frac{1}{2}\right]^{2}\right)}\left|j_{\tau}\right|^{2}+|j|^{2} d s d t \lesssim E^{\frac{1}{2}} .
\end{gathered}
$$


The characterization of the $H^{-1}$-norm implies the existence of $\rho_{1}, \rho_{2}: \mathbb{T}^{2} \rightarrow \mathbb{R}$ such that

$$
\begin{aligned}
& \partial_{1} \tilde{\chi}_{1}-\partial_{2}\left(f_{(100)} \tilde{\chi}_{1}\right)=\partial_{1} \rho_{1}+\partial_{2} \rho_{2} \text { in } \mathcal{D}^{\prime} \\
& \int_{\mathbb{T}^{2}} \rho_{1}^{2}+\rho_{2}^{2} d x \lesssim E^{\frac{1}{2}} .
\end{aligned}
$$

Due to the structure of the change of coordinates $\Phi$, a distributional chain rule holds:

$$
\partial_{s}\left(\tilde{\chi}_{1} \circ \Phi\right)=\partial_{s}\left(\rho_{\sigma} \circ \Phi\right)+\partial_{t}\left(\rho_{\tau} \circ \Phi\right)
$$

for $\rho_{\sigma}=\rho_{1}$ and $\rho_{\tau}=f_{(100)} \rho_{1}+\rho_{2}$. Consequently we have:

$$
\int_{\Phi^{-1}\left(\mathbb{T}^{2}\right)}\left|\rho_{\sigma}(\Phi(s, t))\right|^{2}+\left|\rho_{\tau}(\Phi(s, t))\right|^{2} d s d t \lesssim E^{\frac{1}{2}} .
$$

As sums and products of $\left[-\frac{1}{2}, \frac{1}{2}\right]^{2}$-periodic functions, $\rho_{\sigma}$ and $\rho_{\tau}$ are periodic as well. A calculation as in Lemma 2$]$ converts this into

$$
\begin{aligned}
\partial_{s}^{h}\left(\left(\tilde{\chi}_{1} \circ \Phi\right)-\left(\rho_{\sigma} \circ \Phi\right)\right)(s, t) & =\int_{0}^{h} \frac{d}{d h^{\prime}}\left(\left(\tilde{\chi}_{1} \circ \Phi\right)-\left(\rho_{\sigma} \circ \Phi\right)\right)\left(s+h^{\prime}, t\right) d h^{\prime} \\
& =\frac{d}{d t} \int_{0}^{h} \rho_{\tau}\left(\Phi\left(s+h^{\prime}, t\right)\right) d h^{\prime} .
\end{aligned}
$$

Setting

$$
\begin{aligned}
& j(s, t):=\partial_{s}^{h} \rho_{\sigma}(\Phi(s, t)), \\
& j_{\tau}(s, t):=\int_{0}^{h} \rho_{\tau}\left(\Phi\left(s+h^{\prime}, t\right)\right) d h^{\prime},
\end{aligned}
$$

and using (18), we obtain

$$
\begin{gathered}
\partial_{s}^{h} \tilde{\chi}_{1}(\Phi(s, t))=\partial_{t} j_{\tau}(s, t)+j(s, t) \\
\int_{\Phi^{-1}\left(\left[-\frac{1}{2}, \frac{1}{2}\right]^{2}\right)}\left|j_{\tau}\right|^{2}+|j|^{2} d s d t \lesssim E^{\frac{1}{2}}
\end{gathered}
$$

Step 3: Interpolation inequality.

We use the interpolation inequality of Lemma 3 applied to (the periodic function) $\phi=\left(\partial_{s}^{h}\left(\tilde{\chi}_{1} \circ \Phi\right)\right) \circ \Phi^{-1}$ in its multiplicative version and carry out a change of coordinates 
to derive:

$$
\begin{aligned}
\int_{\Phi^{-1}\left(\mathbb{T}^{2}\right)}\left|\partial_{s}^{h} \tilde{\chi}_{1}(\Phi(s, t))\right|^{2} d s d t & \\
\leq & \left(\int_{\Phi^{-1}\left(\mathbb{T}^{2}\right)}\left|\nabla_{(s, t)}\left(\partial_{s}^{h} \tilde{\chi}_{1}(\Phi(s, t))\right)\right| d s d t \sup \left|\left(\partial_{s}^{h}\left(\tilde{\chi}_{1} \circ \Phi\right)\right) \circ \Phi^{-1}\right|\right)^{\frac{2}{3}} \\
& \times\left(\int_{\Phi^{-1}\left(\mathbb{T}^{2}\right)}\left|j_{\tau}\right|^{2}+|j|^{2} d s d t\right)^{\frac{1}{3}}
\end{aligned}
$$

More precisely, the multiplicative version is first used on the torus with the periodic functions

$$
\left(\partial_{s}^{h}\left(\tilde{\chi}_{1} \circ \Phi\right)\right) \circ \Phi^{-1}, j \circ \Phi^{-1}, j_{\tau} \circ \Phi^{-1} .
$$

In a second step the transformation rule is applied together with the volume conservation of $\Phi$ which then yields the desired result.

In order to convince oneself of the periodicity of $\left(\partial_{s}^{h}\left(\tilde{\chi}_{1} \circ \Phi\right)\right) \circ \Phi^{-1}, j \circ \Phi^{-1}, j_{\tau} \circ \Phi^{-1}$ we argue by inserting the change of coordinates:

$$
\begin{aligned}
\left(\left(\partial_{s}^{h}\left(\tilde{\chi}_{1} \circ \Phi\right)\right) \circ \Phi^{-1}\right)\left(y_{1}, y_{2}\right)= & \tilde{\chi}_{1}\left(y_{1}+h, y_{2}-F_{(100)}\left(y_{1}+h\right)+F_{(100)}\left(y_{1}\right)\right) \\
& -\tilde{\chi}_{1}\left(y_{1}, y_{2}\right) .
\end{aligned}
$$

Here $F_{(100)}$ denotes the normalized antiderivative of $f_{(100)}$ (i.e. $\left.F_{(100)}(0)=0\right)$. Since $f_{(100)}$ is the mean value of a periodic function it is periodic itself. Hence, the fundamental theorem of calculus implies that $F_{(100)}\left(y_{1}+h\right)-F_{(100)}\left(y_{1}\right)$ inherits this property. Combined with the periodicity of $\tilde{\chi}_{1}$ this implies the periodicity of $\left(\partial_{s}^{h}\left(\tilde{\chi}_{1} \circ \Phi\right)\right) \circ \Phi^{-1}$. The periodicity of $j \circ \Phi^{-1}$ follows from the periodicity of $\rho_{\sigma}$.

A similar argument works for $j_{\tau} \circ \Phi^{-1}$ : Due to

$$
\left(j_{\tau} \circ \Phi^{-1}\right)\left(y_{1}, y_{2}\right)=\int_{0}^{h} \rho_{\tau}\left(y_{1}+h^{\prime}, y_{2}-F_{(100)}\left(y_{1}+h^{\prime}\right)+F_{(100)}\left(y_{1}\right)\right) d h^{\prime},
$$

the periodicity of $F_{(100)}\left(y_{1}+h^{\prime}\right)-F_{(100)}\left(y_{1}\right)$ and $\rho_{\tau}$ implies the claim.

Using an approximation argument the first term in the interpolation inequality can be estimated by the homogeneous BV norm of the martensitic phases. Together with the 
$L^{\infty}$-bound for $\tilde{\chi}_{1}$, this yields the desired result:

$$
\begin{aligned}
\int_{\Phi^{-1}\left(\mathbb{T}^{2}\right)}\left|\partial_{s}^{h} \tilde{\chi}_{1}(\Phi(s, t))\right|^{2} d s d t & \\
\leq & \left(\int_{\Phi^{-1}\left(\mathbb{T}^{2}\right)}\left|\nabla_{(s, t)}\left(\partial_{s}^{h} \tilde{\chi}_{1}(\Phi(s, t))\right)\right| d s d t \sup \left|\left(\partial_{s}^{h}\left(\tilde{\chi}_{1} \circ \Phi\right)\right) \circ \Phi^{-1}\right|\right)^{\frac{2}{3}} \\
& \times\left(\int_{\Phi^{-1}\left(\mathbb{T}^{2}\right)}\left|j_{\tau}\right|^{2}+|j|^{2} d s d t\right)^{\frac{1}{3}} \\
& \left(\int_{\mathbb{T}^{2}}\left|\nabla \chi_{1}\right|+\left|\nabla \chi_{4}\right| d x\right)^{\frac{2}{3}}\left(\int_{\Phi^{-1}\left(\mathbb{T}^{2}\right)}\left|j_{\tau}\right|^{2}+|j|^{2} d s d t\right)^{\frac{1}{3}} \\
\lesssim & \left(E_{\text {surf }}\right)^{\frac{2}{3}} E^{\frac{1}{6}} \lesssim\left(\eta^{-\frac{1}{3}} E\right)^{\frac{2}{3}} E^{\frac{1}{6}} \lesssim \eta^{-\frac{2}{9}} E^{\frac{5}{6}} \cdot
\end{aligned}
$$

Finally, the $L^{1}$-estimate follows from the discreteness of $\tilde{\chi}_{1}$.

Step 4: $L^{1}$-control.

There exists $g: \Phi^{-1}\left(\left[-\frac{1}{2}, \frac{1}{2}\right]^{2}\right) \rightarrow \mathbb{R},(s, t) \mapsto g(t)$ such that

$$
\begin{gathered}
\int_{\Phi^{-1}\left(\left[-\frac{1}{2}, \frac{1}{2}\right]^{2}\right)}\left|\tilde{\chi}_{1}(\Phi(s, t))-g(t)\right| d s d t \\
\lesssim \sup _{|h| \leq 1} \int_{\Phi^{-1}\left(\left[-\frac{1}{2}, \frac{1}{2}\right]^{2}\right)}\left|\partial_{s}^{h} \tilde{\chi}_{1}(\Phi(s, t))\right| d s d t \lesssim \eta^{-\frac{2}{9}} E^{\frac{5}{6}} .
\end{gathered}
$$

As $\Phi$ is a bilipschitz mapping, we find $r \geq 1$ and $k \in \mathbb{N}, k \geq 2$ such that the following inclusions are satisfied

$$
\Phi^{-1}\left(\left[-\frac{1}{2}, \frac{1}{2}\right]^{2}\right) \subset\left[-\frac{r}{2}, \frac{r}{2}\right]^{2} \subset[-r, r]^{2} \subset \Phi^{-1}\left(\left[-\frac{k}{2}, \frac{k}{2}\right]^{2}\right)
$$


Thus, the claim is a consequence of the following estimates:

$$
\begin{aligned}
\sup _{|h| \leq 1} \int_{\Phi^{-1}\left(\left[-\frac{k}{2}, \frac{k}{2}\right]^{2}\right)} \mid \tilde{\chi}_{1}(\Phi(s+h, t)) & -\tilde{\chi}_{1}(\Phi(s, t)) \mid d s d t \\
& \geq \frac{1}{2} \int_{-1}^{1} \int_{[-r, r]^{2}}\left|\tilde{\chi}_{1}(\Phi(s+h, t))-\tilde{\chi}_{1}(\Phi(s, t))\right| d s d t d h \\
& \gtrsim \int_{\left[-\frac{r}{2}, \frac{r}{2}\right]^{2}-\frac{1}{2}}^{\frac{1}{2}}\left|\tilde{\chi}_{1}(\Phi(y, t))-\tilde{\chi}_{1}(\Phi(s, t))\right| d y d s d t \\
& \gtrsim \int_{\left[-\frac{r}{2}, \frac{r}{2}\right]^{2}}\left|\tilde{\chi}_{1}(\Phi(s, t))-\int_{-\frac{1}{2}}^{\frac{1}{2}} \tilde{\chi}_{1}(\Phi(y, t)) d y\right| d s d t \\
& \int_{\Phi^{-1}\left(\left[-\frac{1}{2}, \frac{1}{2}\right]^{2}\right)}\left|\tilde{\chi}_{1}(\Phi(s, t))-g(t)\right| d s d t,
\end{aligned}
$$

where $g(t)=\int_{-\frac{1}{2}}^{\frac{1}{2}} \tilde{\chi}_{1}(\Phi(y, t)) d y$.

Since

$$
\begin{aligned}
& \sup _{|h| \leq 1} \int_{\Phi^{-1}\left(\left[-\frac{k}{2}, \frac{k}{2}\right]^{2}\right)}\left|\partial_{s}^{h} \tilde{\chi}_{1}(\Phi(s, t))\right| d s d t \\
& \leq C k^{2} \sup _{|h| \leq 1} \int_{\Phi^{-1}\left(\left[-\frac{1}{2}, \frac{1}{2}\right]^{2}\right)}\left|\partial_{s}^{h} \tilde{\chi}_{1}(\Phi(s, t))\right| d s d t \lesssim \eta^{-\frac{2}{9}} E^{\frac{5}{6}}
\end{aligned}
$$

the claim is proven in the $L^{1}$-topology.

Due to the discreteness/boundedness of all quantities, we also obtain the $L^{2}$-estimate for $\tilde{\chi}_{1}$.

Step 5: Estimate for $\tilde{\chi}_{2}$.

Let $g: \Phi^{-1}\left(\left[-\frac{1}{2}, \frac{1}{2}\right]^{2}\right) \rightarrow \mathbb{R}$ be the function from step 4 . The estimate for $\tilde{\chi}_{1}$ in 
combination with the identity $\tilde{\chi}_{2}=\tilde{\chi}_{3} \tilde{\chi}_{1}$ yields:

$$
\begin{aligned}
&\left\|\tilde{\chi}_{2} \circ \Phi-\left(f_{(100)} \circ \Phi\right) g\right\|_{L^{2}\left(\Phi^{-1}\left(\left[-\frac{1}{2}, \frac{1}{2}\right]^{2}\right)\right)} \\
&=\left\|\left(\tilde{\chi}_{3} \circ \Phi\right)\left(\tilde{\chi}_{1} \circ \Phi\right)-\left(f_{(100)} \circ \Phi\right) g\right\|_{L^{2}\left(\Phi^{-1}\left(\left[-\frac{1}{2}, \frac{1}{2}\right]^{2}\right)\right)} \\
& \leq\left\|\left(\tilde{\chi}_{3} \circ \Phi\right)\left(\left(\tilde{\chi}_{1} \circ \Phi\right)-g\right)\right\|_{L^{2}\left(\Phi^{-1}\left(\left[-\frac{1}{2}, \frac{1}{2}\right]^{2}\right)\right)} \\
& \quad+\left\|g\left(\left(f_{(100)} \circ \Phi\right)-\tilde{\chi}_{3} \circ \Phi\right)\right\|_{L^{2}\left(\Phi^{-1}\left(\left[-\frac{1}{2}, \frac{1}{2}\right]^{2}\right)\right)} \\
& \leq\left\|\tilde{\chi}_{3} \circ \Phi\right\|_{\left.L^{\infty}\left(\Phi^{-1}\left(\left[-\frac{1}{2}, \frac{1}{2}\right]^{2}\right)\right)\right)}\left\|\tilde{\chi}_{1} \circ \Phi-g\right\|_{L^{2}\left(\Phi^{-1}\left(\left[-\frac{1}{2}, \frac{1}{2}\right]^{2}\right)\right)} \\
& \quad+\|g\|_{\left.\left.L^{\infty}\left(\Phi^{-1}\left(\left[-\frac{1}{2}, \frac{1}{2}\right]^{2}\right)\right)\right)\right)}\left\|\left(f_{(100)} \circ \Phi\right)-\tilde{\chi}_{3} \circ \Phi\right\|_{L^{2}\left(\Phi^{-1}\left(\left[-\frac{1}{2}, \frac{1}{2}\right]^{2}\right)\right)} \\
& \lesssim \eta^{-\frac{1}{9}} E^{\frac{5}{12}}+E^{\frac{1}{4}} .
\end{aligned}
$$

This proves the claim for $\tilde{\chi}_{2}$.

Finally, we present the counterexample stating that we have to use the BV control in order to obtain strong rigidity.

Lemma 7. Let $f_{(100)}: \mathbb{T}^{2} \rightarrow\{-1,1\},(s, t) \mapsto f_{(100)}(s)$. Then there exists a sequence $\left\{\left(\tilde{\chi}_{1}^{(k)}, \tilde{\chi}_{2}^{(k)}, \tilde{\chi}_{3}^{(k)}\right)\right\}_{k \in \mathbb{N}}, \tilde{\chi}_{i}^{(k)}: \mathbb{T}^{2} \rightarrow\{-1,1\}$, and corresponding sequences $\left\{u^{(k)}\right\}_{k \in \mathbb{N}}$, $u^{(k)}: \mathbb{T}^{2} \rightarrow \mathbb{R}$ and $\left\{g^{(k)}\right\}_{k \in \mathbb{N}}, g^{(k)}: \mathbb{T}^{2} \rightarrow \mathbb{R},(s, t) \mapsto g^{(k)}(t)$ such that

$$
\begin{aligned}
& \left\|\left(\begin{array}{c}
\tilde{\chi}_{2}^{(k)}-f_{(100)} \tilde{\chi}_{1}^{(k)} \\
\tilde{\chi}_{1}^{(k)}
\end{array}\right)-\left(\begin{array}{c}
\partial_{s} u^{(k)} \\
\partial_{t} u^{(k)}
\end{array}\right)\right\|_{L^{2}\left(\mathbb{T}^{2}\right)} \leq \frac{1}{k}, \\
& \left\|u^{(k)}-g^{(k)}\right\|_{L^{2}\left(\mathbb{T}^{2}\right)} \leq \frac{1}{k} \\
& \left\|\partial_{s} u^{(k)}\right\|_{L^{2}\left(\mathbb{T}^{2}\right)} \leq \frac{1}{k} \\
& \tilde{\chi}_{2}^{(k)}-\tilde{\chi}_{3}^{(k)} \tilde{\chi}_{1}^{(k)}=0 \\
& \tilde{\chi}_{3}^{(k)}=f_{(100)},
\end{aligned}
$$

but

$$
\left\|\tilde{\chi}_{1}^{(k)}-f\right\|_{L^{2}\left(\mathbb{T}^{2}\right)} \geq C
$$

for all $f: \mathbb{T}^{2} \rightarrow \mathbb{R},(s, t) \mapsto f(t)$. Here $C>0$ is a universal constant.

Proof. In order to define the sequence $\tilde{\chi}_{i}^{(k)}$, we first construct a sample which is then rescaled appropriately. For that purpose, consider the function $u: \mathbb{T}^{2} \rightarrow \mathbb{R}$, whose gradient is depicted in Figure 2 on the interval $\left[-\frac{1}{2}, \frac{1}{2}\right]^{2}$ and for which we have $u=0$ on $\{s=t\} \cap\left[-\frac{1}{2}, 0\right]^{2}$ and on $\{t=-s\} \cap\left\{\left[0, \frac{1}{2}\right] \times\left[-\frac{1}{2}, 0\right]\right\}$. This is to be continued periodically. 


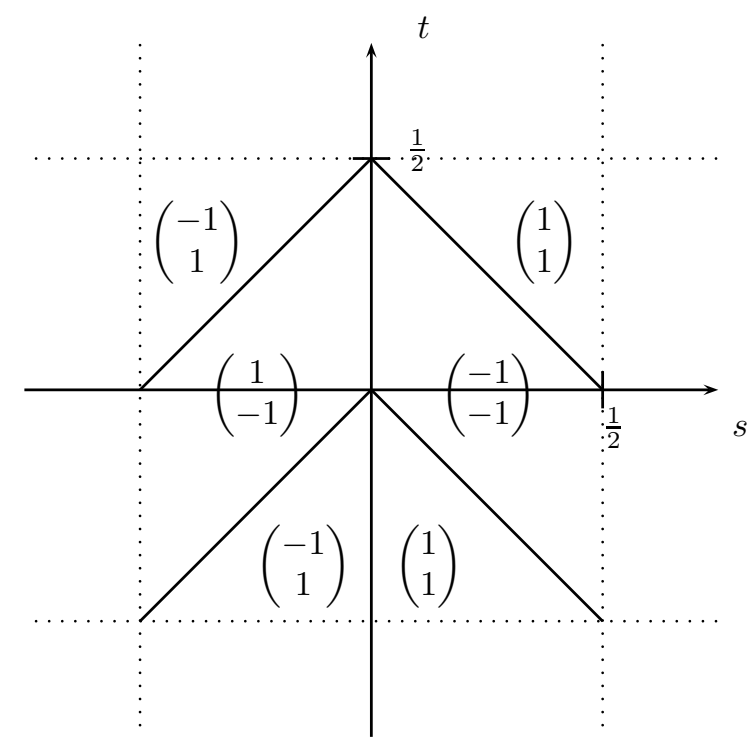

Figure 2: On the torus $\partial_{s} u$ is depicted in the first and $\partial_{t} u$ in the second component.

On $\left[-\frac{1}{2}, \frac{1}{2}\right] \times\left[-\frac{1}{4},-\frac{3}{8}\right]$ the $s$-average of the $t$-derivative is given by

$$
\int_{-\frac{1}{2}}^{\frac{1}{2}} \partial_{t} u(s, \cdot) d s=-4 t-1 .
$$

Thus, the deviation from the average can be estimated by

$$
\begin{aligned}
\int_{\left[-\frac{1}{2}, \frac{1}{2}\right]^{2}}\left|\partial_{t} u-\int_{-\frac{1}{2}}^{\frac{1}{2}} \partial_{t} u\left(s^{\prime}, \cdot\right) d s^{\prime}\right|^{2} d s d t & \geq \int_{\left[-\frac{1}{4}, \frac{1}{4}\right] \times\left[-\frac{1}{4},-\frac{3}{8}\right]}\left|1+4\left(t+\frac{1}{4}\right)\right|^{2} d s d t \\
& \geq \frac{1}{2} \cdot \frac{1}{8} \cdot \frac{1}{4}=\frac{1}{64}>0 .
\end{aligned}
$$

We rescale and set

$$
u^{(k)}(s, t):=\frac{1}{k^{2}} u\left(k s, k^{2} t\right), k \in \mathbb{N} .
$$

Further we define

$$
\begin{aligned}
& \tilde{\chi}_{1}^{(k)}:=\partial_{t} u^{(k)} \in\{-1,1\}, \\
& \tilde{\chi}_{2}^{(k)}:=f_{(100)} \partial_{t} u^{(k)} \in\{-1,1\}, \\
& \tilde{\chi}_{3}^{(k)}:=f_{(100)} .
\end{aligned}
$$


Hence, (19), (20), (21), (22) are satisfied since

$$
\left\|\left(\begin{array}{c}
\tilde{\chi}_{2}^{(k)}-f_{(100)} \tilde{\chi}_{1}^{(k)} \\
\tilde{\chi}_{1}^{(k)}
\end{array}\right)-\left(\begin{array}{c}
\partial_{s} u^{(k)} \\
\partial_{t} u^{(k)}
\end{array}\right)\right\|_{L^{2}\left(\mathbb{T}^{2}\right)}=\left\|\partial_{s} u^{(k)}\right\|_{L^{2}\left(\mathbb{T}^{2}\right)} \leq \frac{1}{k},
$$

and since due to $|u| \leq \frac{1}{2}$, we have $\left|u^{(k)}\right| \leq \frac{1}{k^{2}}$. Therefore, $\left\|u^{(k)}\right\|_{L^{2}\left(\mathbb{T}^{2}\right)} \leq \frac{1}{k^{2}}$. Due to (24), we obtain

$$
\int_{\left[-\frac{1}{2}, \frac{1}{2}\right]^{2}}\left|\tilde{\chi}_{1}^{(k)}-\int_{-\frac{1}{2}}^{\frac{1}{2}} \tilde{\chi}_{1}^{(k)}\left(s^{\prime}, \cdot\right) d s^{\prime}\right|^{2} d s d t \geq \frac{1}{64} .
$$

Finally, this leads to

$$
\inf _{f(t) \in L^{2}\left(\left[-\frac{1}{2}, \frac{1}{2}\right]\right)}\left\|\tilde{\chi}_{1}^{(k)}-f\right\|_{L^{2}\left(\mathbb{T}^{2}\right)}^{2} \geq \frac{1}{64}>0,
$$

as taking the mean value corresponds to the $L^{2}$-projection on the space of constants.

\subsection{Optimality and Branching}

In this final section we give an upper bound construction to derive the optimal scaling behavior of incompatible microstructures. Combined with Theorem 2 , this proves Proposition 1, The construction relies on an idea introduced in [2].

Lemma 8. Let $\eta \ll 1$, consider

$$
\begin{gathered}
E_{\eta}(e, \chi):=\eta^{\frac{1}{3}} \int_{(0,1)^{2}}\left|\nabla \chi_{1}\right|+\left|\nabla \chi_{2}\right|+\left|\nabla \chi_{3}\right|+\left|\nabla \chi_{4}\right| d y \\
\quad+\eta^{-\frac{2}{3}} \int_{(0,1)^{2}}\left|e-\left(\begin{array}{ccc}
d_{1} & \tilde{\chi}_{3} & \tilde{\chi}_{2} \\
\tilde{\chi}_{3} & d_{2} & \tilde{\chi}_{1} \\
\tilde{\chi}_{2} & \tilde{\chi}_{1} & d_{3}
\end{array}\right)\right|^{2} d y .
\end{gathered}
$$

For $\delta=\frac{3}{16}$ there exists a family of $(0,1)^{2}$-periodic strain tensors $\left\{e_{\eta}\right\}_{\eta} \in \operatorname{Sym}(3, \mathbb{R})$, $e_{\eta}=e_{\eta}\left(y_{1}, y_{2}\right)$, and a family $\left\{\chi^{\eta}=\left(\tilde{\chi}_{2}^{\eta}, \tilde{\chi}_{3}^{\eta}, \tilde{\chi}_{1}^{\eta}\right)\right\}_{\eta}, \tilde{\chi}_{i} \in\{-1,1\}$ such that

$$
E_{\eta}\left(e_{\eta}, \chi^{\eta}\right) \lesssim 1
$$

and

$$
\begin{aligned}
& \left|\theta_{1}^{\eta}\left(\theta_{2}^{\eta}+\theta_{4}^{\eta}\right)-\theta_{4}^{\eta}\left(\theta_{1}^{\eta}+\theta_{3}^{\eta}\right)\right| \geq \delta \\
& \left|\theta_{1}^{\eta}\left(\theta_{2}^{\eta}+\theta_{4}^{\eta}\right)-\theta_{2}^{\eta}\left(\theta_{1}^{\eta}+\theta_{3}^{\eta}\right)\right| \geq \delta .
\end{aligned}
$$


Proof. Step 1: Choice of $e_{\eta}$.

Consider

$$
\begin{aligned}
E_{\eta}(\chi):= & \eta^{\frac{1}{3}} \int_{(0,1)^{2}}\left|\nabla \chi_{1}\right|+\left|\nabla \chi_{2}\right|+\left|\nabla \chi_{3}\right|+\left|\nabla \chi_{4}\right| d x \\
& +\eta^{-\frac{2}{3}} \inf _{\nabla u \text { periodic }}\left\{\int_{(0,1)^{2}}\left|\frac{\nabla u+(\nabla u)^{t}}{2}-\left(\begin{array}{ccc}
d_{1} & \tilde{\chi}_{3} & \tilde{\chi}_{2} \\
\tilde{\chi}_{3} & d_{2} & \tilde{\chi}_{1} \\
\tilde{\chi}_{2} & \tilde{\chi}_{1} & d_{3}
\end{array}\right)\right|^{2} d x\right\} \\
= & : \eta^{\frac{1}{3}} E_{\text {surf }}(\chi)+\eta^{-\frac{2}{3}} E_{\text {elast }}(\chi) .
\end{aligned}
$$

For each $\chi$ and each $\eta \in(0,1)$ there exists $e_{\eta}$ such that

$$
\left|E_{\eta}(\chi)-E_{\eta}\left(e_{\eta}, \chi\right)\right| \leq E_{\eta}(\chi)
$$

which especially implies

$$
E_{\eta}\left(e_{\eta}, \chi\right) \leq 2 E_{\eta}(\chi)
$$

Thus, it suffices to estimate $E_{\eta}(\chi)$ in the sequel.

Step 2: Calculation of the Fourier multiplier for the elastic energy.

In order to obtain the Fourier multiplier of the elastic energy the Euler-LagrangeEquations of the elastic energy have to be computed. These are well known (c.f. [2]) and lead to

$$
E_{\text {elast }}(\chi)=\sum_{\substack{k \in \mathbb{Z}^{2} \\ k \neq 0}}|k|^{-4}\left(|k|^{4}\left|\mathcal{F} \tilde{U}_{0}\right|^{2}-2|k|^{2}\left|\mathcal{F} \tilde{U}_{0} k\right|^{2}+\left|k \cdot \mathcal{F} \tilde{U}_{0} k\right|^{2}\right),
$$

where

$$
\tilde{U}_{0}=\left(\begin{array}{ccc}
d_{1} & \tilde{\chi}_{3} & \tilde{\chi}_{2} \\
\tilde{\chi}_{3} & d_{2} & \tilde{\chi}_{1} \\
\tilde{\chi}_{2} & \tilde{\chi}_{1} & d_{3}
\end{array}\right)
$$

As the Fourier multiplier ignores the mode $k=0$, i.e. constants, we can use

$$
U_{0}:=\left(\begin{array}{ccc}
0 & \tilde{\chi}_{3} & \tilde{\chi}_{2} \\
\tilde{\chi}_{3} & 0 & \tilde{\chi}_{1} \\
\tilde{\chi}_{2} & \tilde{\chi}_{1} & 0
\end{array}\right)
$$

instead. Consequently the summands in the multiplier can be computed as

$$
\begin{aligned}
& |k|^{4}\left|\mathcal{F} U_{0}\right|^{2}=2\left(k_{1}^{4}+k_{2}^{4}+2 k_{1}^{2} k_{2}^{2}\right)\left(\left|\mathcal{F} \tilde{\chi}_{2}\right|^{2}+\left|\mathcal{F} \tilde{\chi}_{3}\right|^{2}+\left|\mathcal{F} \tilde{\chi}_{1}\right|^{2}\right), \\
& |k|^{2}\left|\mathcal{F} U_{0} k\right|^{2}=\left(k_{1}^{2}+k_{2}^{2}\right)\left(k_{1}^{2}\left|\mathcal{F} \tilde{\chi}_{2}\right|^{2}+\left(k_{1}^{2}+k_{2}^{2}\right)\left|\mathcal{F} \tilde{\chi}_{3}\right|^{2}\right. \\
& \left.\quad+k_{2}^{2}\left|\mathcal{F} \tilde{\chi}_{1}\right|^{2}+2 \operatorname{Re}\left(k_{1} \overline{\mathcal{F}} \tilde{\chi}_{2} k_{2} \mathcal{F} \tilde{\chi}_{1}\right)\right), \\
& \left(k \cdot \mathcal{F} U_{0} k\right)^{2}=4 k_{1}^{2} k_{2}^{2}\left|\mathcal{F} \tilde{\chi}_{3}\right|^{2} .
\end{aligned}
$$


Therefore the multiplier is determined by the following expressions:

$$
\begin{array}{rl}
|k|^{-4} & 2\left(k_{1}^{4}\left|\mathcal{F} \tilde{\chi}_{1}\right|^{2}+k_{2}^{4}\left|\mathcal{F} \tilde{\chi}_{2}\right|^{2}+k_{1}^{2} k_{2}^{2}\left|\mathcal{F} \tilde{\chi}_{2}\right|^{2}+2 k_{1}^{2} k_{2}^{2}\left|\mathcal{F} \tilde{\chi}_{3}\right|^{2}+k_{1}^{2} k_{2}^{2}\left|\mathcal{F} \tilde{\chi}_{1}\right|^{2}\right. \\
& \left.-2 k_{1}^{2} \operatorname{Re}\left(k_{1} \overline{\mathcal{F}} \tilde{\chi}_{2} k_{2} \mathcal{F} \tilde{\chi}_{1}\right)-2 k_{2}^{2} \operatorname{Re}\left(k_{1} \overline{\mathcal{F}} \tilde{\chi}_{2} k_{2} \mathcal{F} \tilde{\chi}_{1}\right)\right) \\
& =2|k|^{-4}\left(|k|^{2}\left|k_{2} \mathcal{F} \tilde{\chi}_{2}-k_{1} \mathcal{F} \tilde{\chi}_{1}\right|^{2}+2 k_{1}^{2} k_{2}^{2}\left|\mathcal{F} \tilde{\chi}_{3}\right|^{2}\right) .
\end{array}
$$

Step 3: Introduction of the quantities involved in the construction. For $\lambda \in[0,1],\left(y_{1}, y_{2}\right) \in[0,1]^{2}$ we define $\sigma_{(\lambda, 1)}^{\eta}\left(y_{1}, y_{2}\right)$ with

$$
\sigma_{(\lambda, 1)}^{\eta}\left(y_{1}, y_{2}\right):= \begin{cases}\in\{-1,1\}, & y_{2} \in(\lambda, 1) \\ =1-2 \mu, & \text { else. }\end{cases}
$$

For this purpose, we consider the following quantities:

$$
\begin{aligned}
& \mu, \lambda \in[0,1], \\
& N, w_{1}^{-1} \in \mathbb{N}, \\
& w_{n}:=2^{-(n-1)} w_{1}, \\
& l_{n}:=2^{-\beta(n-1)} l_{1}, \beta>0, n \in\{1, \cdots, N\},
\end{aligned}
$$

where we choose $l_{1}$ such that $\sum_{n=1}^{N} l_{n}=\frac{1-\lambda}{2}$. On $[0,1] \times[\lambda, 1]$ we introduce a construction of rows of self-similar cells of width $w_{n}$ and height $l_{n}$ as depicted in Figure 3. Within each of the cells we define $\sigma_{(\lambda, 1)}^{\eta}$ as indicated in Figure 4 .

With this we can define the modified phase functions:

$$
\begin{aligned}
& \quad \tilde{\chi}_{1}^{\eta}:=2 \chi_{(\lambda, 1)}\left(y_{2}\right)-1, \\
& \tilde{\chi}_{2}^{\eta}:=(1-2 \mu)-\sigma_{(0, \lambda)}^{\eta}\left(y_{1}, y_{2}\right)-\sigma_{(\lambda, 1)}^{\eta}\left(y_{1}, y_{2}\right), \\
& \tilde{\chi}_{3}^{\eta}:=\sigma_{(0, \lambda)}^{\eta}\left(y_{1}, y_{2}\right)-(1-2 \mu) \chi_{(\lambda, 1)}\left(y_{2}\right)-\sigma_{(\lambda, 1)}^{\eta}\left(y_{1}, y_{2}\right)+(1-2 \mu) \chi_{(0, \lambda)}\left(y_{2}\right), \\
& \text { with } \chi_{(0, \lambda)}\left(y_{2}\right):= \begin{cases}1, & y_{2} \in(0, \lambda) \\
0, & \text { else. }\end{cases}
\end{aligned}
$$

The definition of $\chi_{(\lambda, 1)}$ is to be understood analogously, just as $\sigma_{(0, \lambda)}^{\eta}\left(y_{1}, y_{2}\right)$ is defined in analogy to $\sigma_{(\lambda, 1)}^{\eta}\left(y_{1}, y_{2}\right)$. We have (c.f. Figure[5 (a))

$$
\left(\tilde{\chi}_{1}^{\eta}, \tilde{\chi}_{2}^{\eta}, \tilde{\chi}_{3}^{\eta}\right) \in\{(1,1,1),(1,-1,-1),(-1,1,-1),(-1,-1,1)\}
$$

This shows that $\tilde{\chi}_{1}^{\eta}, \tilde{\chi}_{2}^{\eta}, \tilde{\chi}_{3}^{\eta}$ originate from $\chi_{1}^{\eta}, \chi_{2}^{\eta}, \chi_{3}^{\eta}, \chi_{4}^{\eta} \in\{0,1\}$, i.e. these functions are indeed modified characteristic functions.

Step 4: Energy estimates.

In oder to estimate the elastic energy, we first remark that via the triangle inequality $\tilde{\chi}_{1}^{\eta}, \tilde{\chi}_{2}^{\eta}, \tilde{\chi}_{3}^{\eta}$ can be decomposed into $\sigma_{(0, \lambda)}^{\eta}, \sigma_{(\lambda, 1)}^{\eta}, \chi_{(0, \lambda)}, \chi_{(\lambda, 1)}$. Since $\chi_{(0, \lambda)}, \chi_{(\lambda, 1)}$ only 


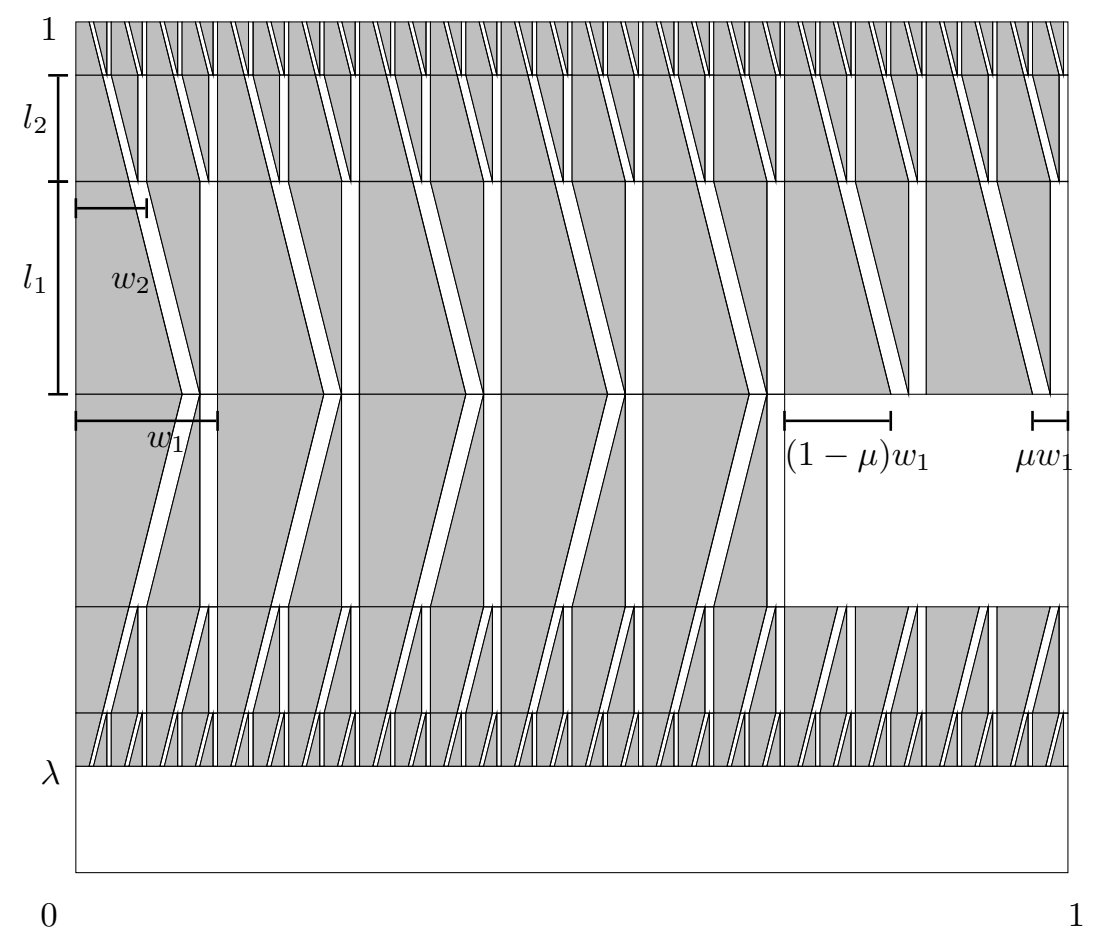

Figure 3: Construction for $\sigma_{(\lambda, 1)}^{\eta}$

depend on $y_{2}$, the terms involving $\mathcal{F} \chi_{(0, \lambda)}, \mathcal{F} \chi_{(\lambda, 1)}$ do not contribute for $k_{1} \neq 0$. As these expressions only occur in the definition of $\tilde{\chi}_{3}, \tilde{\chi}_{1}$ and are therefore multiplied by $k_{1}$, these functions do not play a role for the estimate of the elastic energy.

Consequently, we can conclude:

$$
E_{\text {elast }}\left(\chi^{\eta}\right) \lesssim \sum_{\substack{k \in \mathbb{Z}^{2} \\ k \neq 0}}|k|^{-2} k_{2}^{2}\left(\left|\mathcal{F} \sigma_{(\lambda, 1)}^{\eta}\left(k_{1}, k_{2}\right)\right|^{2}+\left|\mathcal{F} \sigma_{(0, \lambda)}^{\eta}\left(k_{1}, k_{2}\right)\right|^{2}\right) .
$$

With the previous steps we can now reason as in [2]:

Step 5: Estimate of the energy contributions originating from $\sigma_{(\lambda, 1)}^{\eta}$.

We define

$$
\begin{aligned}
& E\left(\sigma_{(\lambda, 1)}^{\eta}\right):=\eta^{-\frac{2}{3}} \sum_{\substack{k \in \mathbb{Z}^{2} \\
k \neq 0}}|k|^{-2} k_{2}^{2}\left|\mathcal{F} \sigma_{(\lambda, 1)}^{\eta}\right|^{2}+\eta^{\frac{1}{3}} \int_{(0,1)^{2}}\left|\nabla \sigma_{(\lambda, 1)}^{\eta}\right| d x \\
& =: \eta^{-\frac{2}{3}} E_{\text {elast }}\left(\sigma_{(\lambda, 1)}^{\eta}\right)+\eta^{\frac{1}{3}} E_{\text {surf }}\left(\sigma_{(\lambda, 1)}^{\eta}\right) .
\end{aligned}
$$

Step 5a: Estimate of the energy within the branching region. 
We consider the following equivalent formulations of the elastic energy:

$$
\begin{gathered}
E_{\text {elast }}\left(\sigma_{(\lambda, 1)}^{\eta}\right)=\sum_{\substack{k \in \mathbb{Z}^{2} \\
k \neq 0}}|k|^{-2} k_{2}^{2}\left|\mathcal{F} \sigma^{\eta}(\lambda, 1)\right|^{2}=\left.\left.\int_{(0,1)^{2}}|| \nabla\right|^{-1} \partial_{2} \sigma_{(\lambda, 1)}^{\eta}\right|^{2} d y \\
=\inf \left\{\int_{(0,1)^{2}}|h|^{2} d y ; h(0,1)^{2} \text {-periodic, } \int_{(0,1)^{2}} h \cdot \nabla \varphi d y=\int_{(0,1)^{2}} \sigma_{(\lambda, 1)}^{\eta} \partial_{2} \varphi d y\right. \\
\left.\forall \varphi: \mathbb{R}^{2} \rightarrow \mathbb{R}^{2},(0,1)^{2} \text {-periodic }\right\} .
\end{gathered}
$$

Thus, choosing $h=\left(\begin{array}{c}h_{1} \\ 0\end{array}\right)$ as indicated in the sketch (c.f. Figure 4), we obtain an upper bound for the energy.
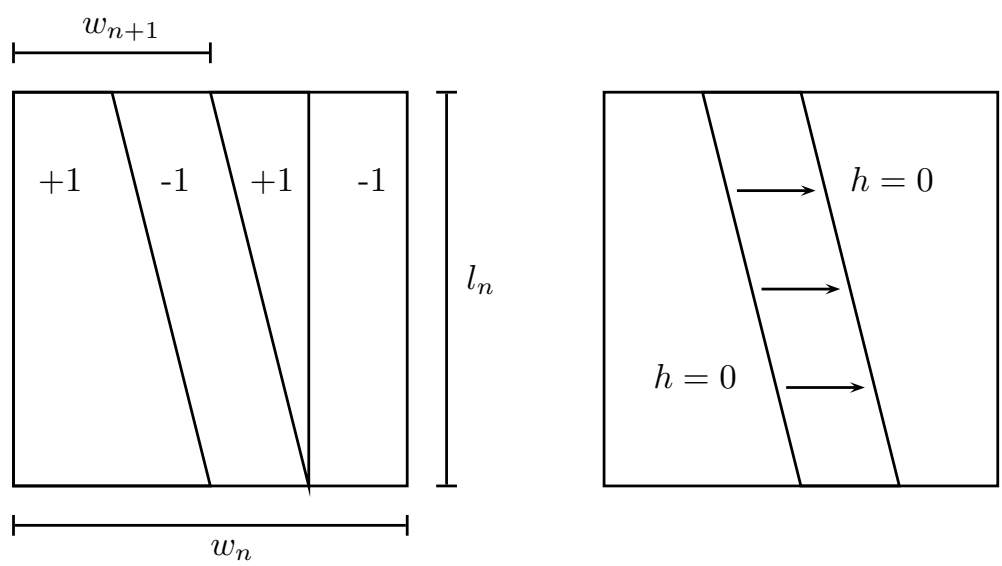

Figure 4: $\sigma_{(\lambda, 1)}^{\eta}$ and the corresponding "field" $h$

Since $|h|=\left|h_{1}\right| \lesssim w_{n} l_{n}^{-1}$ and choosing $w_{n} \leq l_{n}$, we have:

$$
\begin{aligned}
\eta^{\frac{1}{3}} \int\left|\nabla \sigma_{(\lambda, 1)}^{\eta}\right| d y+\eta^{-\frac{2}{3}} \int|h|^{2} d y & \lesssim \eta^{\frac{1}{3}} \max \left\{w_{n}, l_{n}\right\}+\eta^{-\frac{2}{3}}\left(w_{n} l_{n}^{-1}\right)^{2}\left(w_{n} l_{n}\right) \\
& \lesssim \eta^{\frac{1}{3}} l_{n}+\eta^{-\frac{2}{3}} w_{n}^{3} l_{n}^{-1}
\end{aligned}
$$

Summing over the $w_{n}^{-1}$-cells per row, the total energy is bounded by:

$$
\sum_{n=1}^{N} w_{n}^{-1}\left(\eta^{\frac{1}{3}} l_{n}+\eta^{-\frac{2}{3}} w_{n}^{3} l_{n}^{-1}\right)=\sum_{n=1}^{N}\left(w_{n}^{-1} \eta^{\frac{1}{3}} l_{n}+\eta^{-\frac{2}{3}} w_{n}^{2} l_{n}^{-1}\right)
$$

Step 5b: Estimate of the energy within the transition layers.

On $(0,1) \times\left\{y_{2}=\lambda\right\}, \sigma_{(\lambda, 1)}^{\eta}$ jumps from $1-2 \mu$ to 1 and -1 respectively; on $(0,1) \times\left\{y_{2}=\right.$ 
$1\}$ the situation is analogous. Thus, $\partial_{2} \sigma_{(\lambda, 1)}^{\eta}$ displays a singular contribution on these lines. In order to compensate these "charges" we construct a "field" $h$. For this purpose we introduce the transition layers

$$
(0,1) \times\left(\lambda-w_{N+1}, \lambda\right) \cup(0,1) \times\left(1,1+w_{N+1}\right)
$$

where $w_{N+1} \leq \frac{\lambda}{2}$. In oder to secure the permissibility of the "field" $h$ belonging to the $H^{-1}$-norm we have to account for the jumps in direction of the normal. The "field" $h$ is constructed in cells of width $w_{N+1}$ in the first transition layer via a potential $u$ with $h=-\nabla u$. In other words, $u$ is prescribed to satisfy 1

$$
\begin{aligned}
& -\Delta u=0 \text { in }\left(0, w_{N+1}\right) \times\left(\lambda-w_{N+1}, \lambda\right) \\
& \frac{\partial u}{\partial \nu}= \begin{cases}2 \mu & y_{1} \in\left(0,(1-\mu) w_{N+1}\right), y_{2}=\lambda \\
-2(1-\mu) & y_{1} \in\left((1-\mu) w_{N+1}, w_{N+1}\right), y_{2}=\lambda \\
0 & \text { else. }\end{cases}
\end{aligned}
$$

For the second transition layer a similar construction is employed. Thus, we can estimate the elastic energy in the transition layer:

$$
\int_{\left(0, w_{N+1}\right) \times\left(\lambda-w_{N+1}, \lambda\right)}|h|^{2} d y=\int|\nabla u|^{2} d y \lesssim w_{N+1}^{2}
$$

for each of the $w_{N+1}^{-1}$ cells.

Step 5c: Combination of the energy estimates.

Combining the observations of step $5 \mathrm{a}$ and step 5b, we obtain:

$$
\begin{aligned}
E_{\eta}\left(\sigma_{(\lambda, 1)}^{\eta}\right) & =\eta^{\frac{1}{3}} \int\left|\nabla \sigma_{(\lambda, 1)}^{\eta}\right| d y+\eta^{-\frac{2}{3}} \int|h|^{2} d y \\
\lesssim & \sum_{n=1}^{N}\left(\eta^{\frac{1}{3}} w_{n}^{-1} l_{n}+\eta^{-\frac{2}{3}} w_{n}^{2} l_{n}^{-1}\right)+\eta^{\frac{1}{3}}+\eta^{-\frac{2}{3}} w_{N+1} \\
= & \sum_{n=1}^{N}\left(\eta^{\frac{1}{3}} 2^{(n-1)(1-\beta)} \frac{l_{1}}{w_{1}}+\eta^{-\frac{2}{3}} 2^{(n-1)(\beta-2)} \frac{w_{1}^{2}}{l_{1}}\right) \\
& +\eta^{\frac{1}{3}}+2^{-(N+1)} \eta^{-\frac{2}{3}} w_{1} \\
\lesssim & \eta^{\frac{1}{3}} \frac{l_{1}}{w_{1}}+\eta^{-\frac{2}{3}} \frac{w_{1}^{2}}{l_{1}}+\eta^{\frac{1}{3}}+2^{-(N+1)} \eta^{-\frac{2}{3}} w_{1},
\end{aligned}
$$

where we used that, for instance for $\beta=\frac{3}{2}$, we have

$$
\sum_{n=1}^{N} 2^{(n-1)(1-\beta)} \leq C, \quad \sum_{n=1}^{N} 2^{(n-1)(\beta-2)} \leq C .
$$

\footnotetext{
${ }^{1}$ At this point the correct volume fractions for the phases play an essential role as this guarantees that the Neumann problem for the Laplacian can be solved.
} 
Recalling $\frac{1-\lambda}{2} \sim l_{1}$ and minimizing $\eta^{\frac{1}{3}} \frac{1-\lambda}{w_{1}}+\eta^{-\frac{2}{3}} \frac{w_{1}^{2}}{1-\lambda}$ with respect to $w_{1}$, we obtain

$$
w_{1}=\eta^{\frac{1}{3}}(1-\lambda) \text { and } E_{\eta}\left(\chi^{\eta}\right) \lesssim 1+\eta^{\frac{1}{3}}+2^{-(N+1)} \eta^{-\frac{1}{3}} \lesssim 1,
$$

if we choose $N$ so large that $2^{-(N+1)} \lesssim \eta^{\frac{1}{3}}$. Additionally, we have to choose $N$ as to satisfy

$$
\begin{aligned}
& w_{n} \leq l_{n}, \\
& w_{N+1} \leq \frac{\lambda}{2} .
\end{aligned}
$$

For the first condition we define $N$ such that $\eta^{\frac{2}{3}} \leq 2^{-N}$ which can be achieved as $\eta \ll 1$ (which due to $\eta \leq 1$ does not contradict $\left.2^{-(N+1)} \lesssim \eta^{\frac{1}{3}}\right)$. In particular, we can require $\eta^{\frac{2}{3}} \sim 2^{-N}$. Thus, the second condition reduces to $(1-\lambda) \eta \leq \lambda$, which can also be satisfied due to $\eta \ll \lambda$.

Step 6: Conclusion.

Since $\sigma_{(0, \lambda)}^{\eta}$ and $\sigma_{(\lambda, 1)}^{\eta}$ display the same scaling behavior the previous computations lead to the overall energy estimate:

$$
\begin{aligned}
& E_{\eta}\left(\chi^{\eta}\right)=\eta^{\frac{1}{3}} \int\left|\nabla \chi_{1}^{\eta}\right|+\left|\nabla \chi_{2}^{\eta}\right|+\left|\nabla \chi_{3}^{\eta}\right|+\left|\nabla \chi_{4}^{\eta}\right| d x+\eta^{-\frac{2}{3}} E_{\text {elast }} \\
& \quad \lesssim E\left(\sigma_{(0, \lambda)}^{\eta}\right)+E\left(\sigma_{(\lambda, 1)}^{\eta}\right)+\eta^{\frac{1}{3}} \int_{(0,1)^{2}}\left|\nabla \chi_{\left\{y_{2} \in(\lambda, 1)\right\}}\right| d x+\eta^{\frac{1}{3}} \int_{(0,1)^{2}}\left|\nabla \chi_{\left\{y_{2} \in(0, \lambda)\right\}}\right| d x \\
& \quad \lesssim 1+\eta^{\frac{1}{3}} \lesssim 1 .
\end{aligned}
$$

This proves the upper bound.

In order to verify (25), (26), we calculate $\theta_{i}^{\eta}$ as functions of $\lambda$ and of $\mu$. Due to the choice of the phases $\chi_{1}, \ldots, \chi_{4}$ as functions of $\sigma^{\eta}, \chi$ we can easily determine the volume fractions (c.f. Figure 51):

$$
\begin{aligned}
\chi_{1}^{\eta} & =\frac{1}{2}\left(\chi_{(\lambda, 1)}\left(y_{2}\right)-\sigma_{(\lambda, 1)}^{\eta}\left(y_{1}, y_{2}\right)+(1-2 \mu) \chi_{(0, \lambda)}\left(y_{2}\right)\right), \\
\chi_{2}^{\eta} & =\frac{1}{2}\left(1-\chi_{(\lambda, 1)}\left(y_{2}\right)-\sigma_{(0, \lambda)}^{\eta}\left(y_{1}, y_{2}\right)+(1-2 \mu) \chi_{(\lambda, 1)}\left(y_{2}\right)\right), \\
\chi_{3}^{\eta} & =\frac{1}{2}\left(1+\sigma_{(0, \lambda)}^{\eta}\left(y_{1}, y_{2}\right)-(1-2 \mu) \chi_{(\lambda, 1)}\left(y_{1}, y_{2}\right)-\chi_{(\lambda, 1)}\left(y_{2}\right)\right), \\
\chi_{4}^{\eta} & =\frac{1}{2}\left(\sigma_{(\lambda, 1)}^{\eta}\left(y_{1}, y_{2}\right)-(1-2 \mu) \chi_{(0, \lambda)}\left(y_{2}\right)+\chi_{(\lambda, 1)}\left(y_{2}\right)\right) .
\end{aligned}
$$

Thus, we find

$$
\theta_{1}^{\eta}=\mu(1-\lambda), \theta_{2}^{\eta}=\mu \lambda, \theta_{3}^{\eta}=(1-\mu) \lambda, \theta_{4}^{\eta}=(1-\mu)(1-\lambda) .
$$




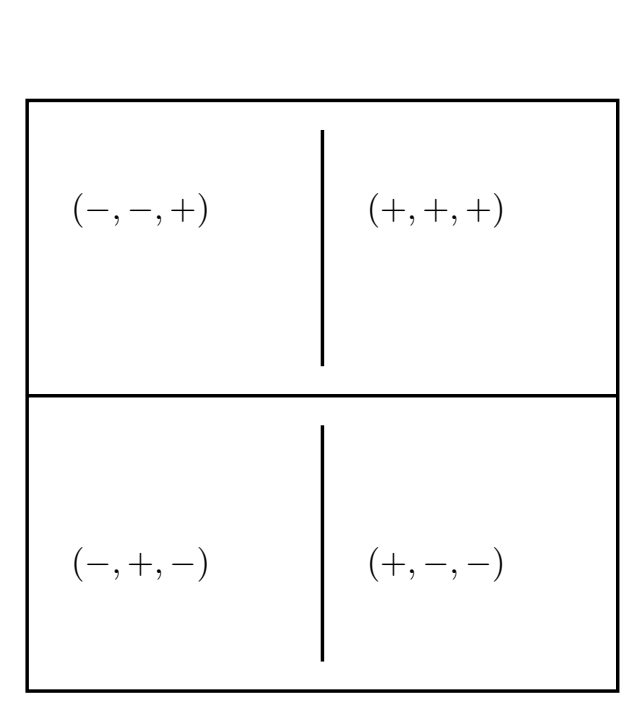

(a)

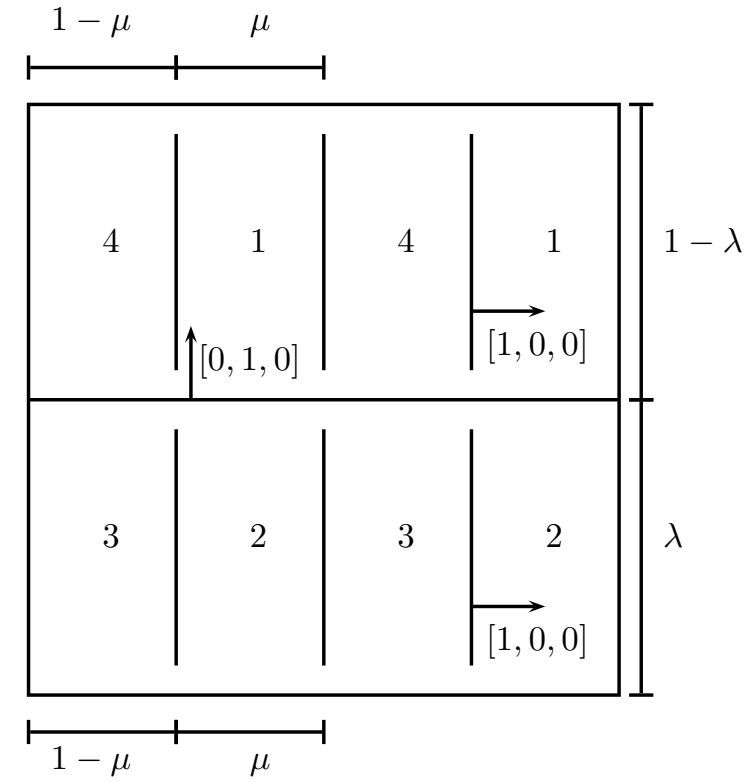

(b)

Figure 5: Schematic arrangement of (a) the modified phase functions $\left(\tilde{\chi}_{2}, \tilde{\chi}_{3}, \tilde{\chi}_{1}\right)$, (b) the actual phase functions $\left(\chi_{1}, \chi_{2}, \chi_{3}, \chi_{4}\right)$

Finally, this yields

$$
\begin{aligned}
& \theta_{1}^{\eta}\left(\theta_{2}^{\eta}+\theta_{4}^{\eta}\right)-\theta_{2}^{\eta}\left(\theta_{1}^{\eta}+\theta_{3}^{\eta}\right)=(1-\mu) \mu(1-2 \lambda), \\
& \theta_{4}^{\eta}\left(\theta_{1}^{\eta}+\theta_{3}^{\eta}\right)-\theta_{1}^{\eta}\left(\theta_{2}^{\eta}+\theta_{4}^{\eta}\right)=\lambda(1-\lambda)(1-2 \mu) .
\end{aligned}
$$

Choosing $\lambda=\mu$, the right hand expression takes values in $\left(0, \frac{3}{16}\right]$ for $\mu \in(0,1)$. As the energy estimate was independent of $\mu, \lambda$, we can choose $\mu, \lambda$ arbitrarily. This implies the claim.

\section{References}

[1] Kaushik Bhatthacharya, microstructure of martensite, why it forms and how it gives rise to the shape-memory effect, New York, Oxford University Press, 2003.

[2] Antonio Capella, Felix Otto, A Rigidity Result for a Perturbation of the Geometrically Linear Three-Well Problem, Bonn, SFB - Preprint, 2008. 
[3] Antonio Capella, Felix Otto, A Quantitative Rigidity Result for the Cubic to Tetragonal Phase Transition in the Geometrically Linear Theory with Interfacial Energy, Bonn, SFB - Preprint, 2010.

[4] Georg Dolzmann, Stefan Müller, The Influence of Surface Energy on Stress-Free Microstructures in Shape Memory Alloys, Meccanica 30: 527-539, 1995.

[5] J.L. Ericksen, The Cauchy Born Hypothesis for crystals, in M.E.Gurtin (ed.) Phase Transformations and Material Instabilities in Solids, pp. 61-78 (Academic Press, 1984)

[6] Lawrence C. Evans, Partial Differential Equations, Providence, AMS, 2002.

[7] Lawrence C. Evans, Ronald F. Gariepy, Measure Theory and Fine Properties of Functions, Boca Raton, CRC Press LLC, 1992

[8] Loukas Grafakos, Classical Fourier Analysis, Berlin, Springer, 2008.

[9] Robert V. Kohn, Stefan Müller, Branching of twins near an austenite-twinnedmartensite interface, Philosophical Magazine A, 1992, Vol 66, No. 5, 697-715

[10] Elliott H. Lieb, Michael Loss, Analysis, Providence, AMS, 2001.

[11] A.E.H. Love, A Treatise on the Mathematical Theory of Elasticity, Dover, 1944.

[12] Stefan Müller, M.A. Sychev, Optimal Existence Theorems in Nonhomogeneous Differential Inclusions, Journal of Functional Analysis 181, 447-475, 2001.

[13] Angkana Rüland, The Cubic-to-Orthorhombic Phase Transition - Rigidity and Non-Rigidity Properties in Linear Elasticity, MPI-MIS Preprint, 45/2013.

[14] Felix Otto, Thomas Viehmann, Domain branching in uniaxial ferromagnets asymptotic behavior of the energy, Bonn, SFB Preprint, (420), 2008.

[15] Mario Pitteri, G. Zanzotto, Continuum Models for Phase Transitions and Twinning in Crystals, Boca Raton, Chapman \& Hall/ CRC Press LLC, 2003 